\title{
The effective description of non-strange hadrons low-energy electro-weak transitions.
}

\author{
G. G. Bunatian * \\ Joint Institute for Nuclear Research, 141980, Dubna, Russia
}

\begin{abstract}
Starting with the general principles of global and local symmetries, the effective pion-nucleon lagrangian, essentially non-linear in pion field, to describe the non-strange hadrons low-energy electro-weak transitions is developed. We encounter no divergence summarizing properly all the infinite power series in pion field which occur in the course of treatment. Our consistent approach proves to be relevant in considering P-parity violation in pion-nucleon interactions.
\end{abstract}

PACS 11.10.Lm Nonlinear or nonlocal theories and models - 11.15.Tk Other nonperturbative techniques - 12.15.Ji Application of electroweak models to specific processes - 11.30.Er Charge conjugation, parity, time reversal, and other discrete symmetries

Keywords: nonlinear pion-nucleon lagrangian; hadron interactions with heavy bosons; P-odd pionnucleon interaction

\section{Introduction}

Nowadays, in actual treating low-energy processes in hadronic systems, in particular the hadron electro-weak transitions, there sees no option but having recourse to the effective lagrangian to describe hadron structure and strong interactions,

$$
\mathcal{L}_{h} \equiv \mathcal{L}_{h}\left[\left\{\phi_{i}(x)\right\},\left\{\partial_{\mu} \phi_{i}(x)\right\}\right],
$$

where the hadron fields $\phi_{i}$ satisfy the Euler-Lagrange equations, and the (anti)commutation relations hold for the Heisenberg field operators $\phi_{i}$ and the corresponding canonically-conjugated momenta (see, for instance, refs. [1-3]).

Plain purpose of the work presented is to study the relevant construction of the effective hadron lagrangian and the respective hadron currents to describe the low-energy electro-weak transitions of nonstrange hadrons. Thus far, the Standard Model $(S M)$ (the Weinberg-Salam theory), which is written

\footnotetext{
${ }^{*}$ Corresponding author.

Email address : bunat@cv.jinr.ru (G. Bunatian)
} 
in terms of quark, gluon, and lepton degrees of freedom, is hardy applied to this problem since we still lack a reliable and calculable way to describe hadrons in terms of their quark-gluon degrees of freedom. Although $Q C D$ is widely understood to be the correct theory of strong interaction, it is difficult to apply rigorously away from the high-energy perturbative area. Since the issue of treating the electro-weak hadronic transitions on the basis of "first principles" is not yet resolved, a calculation starting with the $S M$ involves inevitably simplifying which causes the considerable ambiguities in findings. Our way along, we sidestep plunging into all the intricate $Q C D$ analyzes.

Our treatment is based on the nonlinear realization of the chiral symmetry of the pion-nucleon lagrangian, with this symmetry partially broken. In our consideration, the nucleon and pion are principal particles, and all the other hadronic states, particles and resonances, are interpreted to have the dynamical origin. Although we consider the system of non-strange hadrons stable against strong decay modes, nucleons and pions, the lagrangian $\mathcal{L}_{h}$ is in general certain to describe the variety of electro-weak transitions of non-strange hadrons and hadron resonances, made an expedient allowance for the nucleon-pion strong interaction.

In Sec. II, we treat the essentially nonlinear pion-nucleon lagrangian, with the pion wavefunction and mass renormalization properly carried out. In Appendix A, the dimensional regularization (or continuous dimension) method is considered to treat properly the ultraviolet divergent quantities which occur in our

calculations. In Sec. III, the conserved and partially-conserved hadron currents, radically nonlinear in pion field, associated with that lagrangian are derived. These currents serve to construct, on the local symmetry principle ground, the lagrangian to describe the hadron electro-weak transitions caused by interactions with the electromagnetic field and the intermediate gauge $W^{ \pm}-$and $Z^{0}$ - boson fields, Sec. IV. Upon specifying all the parameters involved in the treatment, this lagrangian is relevant to study the electro-weak processes of significant interest to day. In particular, it provides the way of treating P-parity violation in pion-nucleon interactions, as discussed in Sec. V. In the last Sec. VI, the relevant concluding points are set forth.

The strong interaction is presumed to be adequately allowed for as our treatment is essentially nonlinear in pion field. In our calculation, we are dealing with the infinite power series in pion field. Without restricting by a finite number of terms, we summarize all this infinite series in due course, no divergence emerging thereby, which validates our approach.

\section{Pion-nucleon effective lagrangian}

In order to embark on the path to the effective nonlinear pion-nucleon lagrangian approach, we pursue the method developed in the widely known investigations [4-6]. The nucleon lagrangian to start with,

$$
\mathcal{L}_{\psi}(x)=\bar{\psi}(x)\left[i \gamma^{\mu} \partial_{\mu}-M\right] \psi(x)
$$


is invariant under the global $S U(2)$ isospin rotation

$$
\psi(x) \rightarrow \psi^{\prime}(x)=\mathrm{e}^{-\frac{i}{2} \tau \varepsilon} \psi(x),
$$

where $\left\{\tau_{i}\right\},(i=1,2,3)$ are the Pauli matrices. Yet, as the nucleon mass $M$ is involved, it is apparently non-invariant under the global chiral transformation

$$
\psi(x) \rightarrow \psi^{\prime}(x)=\mathrm{e}^{\frac{i}{2} \gamma^{5} \boldsymbol{a} \boldsymbol{\tau}} \psi(x) .
$$

To balance out the chirality violation, the nucleon interaction with the compensating pseudo scalar field $\varphi(x)$ is introduced by the replacement

$$
\begin{array}{r}
\mathcal{L}_{\psi} \rightarrow \mathcal{L}_{\psi \varphi}=\bar{\psi}\left[i \gamma^{\mu} \partial_{\mu}-M \mathbf{U}\right] \psi=\bar{\psi} i \gamma^{\mu} \partial_{\mu} \psi-\bar{\psi} M \psi+\mathcal{L}_{\psi \varphi}^{i n t}, \\
\mathcal{L}_{\psi \varphi}^{i n t}=M \bar{\psi}(1-\mathbf{U}) \psi,
\end{array}
$$

with $\mathbf{U}$ being a matrix function of $i \gamma^{5} \tau \varphi$. Provided the transformations

$$
\begin{array}{r}
\mathbf{U} \rightarrow \mathbf{U}^{\prime}=\mathrm{e}^{-\frac{i}{2} \varepsilon \tau} \mathrm{Ue}^{\frac{i}{2} \varepsilon \boldsymbol{\tau}}, \\
\mathbf{U} \rightarrow \mathbf{U}^{\prime}=\mathrm{e}^{-\frac{i}{2} \gamma^{5} \boldsymbol{a} \tau} \mathbf{U} \mathrm{e}^{-\frac{i}{2} \gamma^{5} \boldsymbol{a} \boldsymbol{\tau}}
\end{array}
$$

are constrained when the nucleon field transforms by eqs. (3), (4), the lagrangian (5) proves to be invariant under the global $S U(2) \times S U(2)$ isospin and chiral transformation. As we purpose to study the low-energy hadron electro-weak processes, the lagrangian of the pseudo-scalar compensating field $\varphi$ itself is written in the simplest, two-derivative, invariant form

$$
\mathcal{L}_{\varphi \varphi}=\frac{1}{16 f^{2}} \operatorname{Sp}\left[\partial_{\mu} \mathbf{U} \partial^{\mu} \mathbf{U}^{\dagger}\right]
$$

For non-linear realization of the isospin and chiral global invariance of lagrangian, the specific form of the matrix $\mathbf{U}$ is chosen

$$
\mathbf{U}=\mathrm{e}^{-i 2 f \gamma^{5} \tau \varphi}
$$

so that $\mathcal{L}_{\varphi \varphi}(9)$ will reduce to the ordinary pseudo-scalar field kinetic energy

$$
\mathcal{L}_{\varphi \varphi} \approx \frac{1}{2} \partial_{\mu} \varphi \partial^{\mu} \varphi
$$

when the dimensional parameter $f$ tends to zero. The interaction $\mathcal{L}_{\psi \varphi}^{\text {int }}(6)$ reduces in this limit as follows

$$
\mathcal{L}_{\psi \varphi}^{i n t} \approx 2 \text { if } M \bar{\psi} \gamma^{5} \tau \varphi \psi
$$

To associate $\boldsymbol{\varphi}$ with the real pion field of the mass $m$, the lagrangian $\mathcal{L}_{\varphi \varphi}(9)$ is known to be accomplished $[5,6]$ by adding the chiral symmetry-breaking term

$$
\begin{array}{r}
\mathcal{L}_{S B}=\frac{m^{2}}{16 f^{2}} \operatorname{Sp}\left[\mathbf{U}_{\xi}+\mathbf{U}_{\xi}^{\dagger}-2\right]=-\frac{m^{2}}{2 f^{2}} \sin ^{2}\left(f \sqrt{\varphi^{2}}\right) \approx-\frac{m^{2} \varphi^{2}}{2}+\frac{m^{2} f^{2} \varphi^{4}}{6}, \\
\mathbf{U}_{\xi}=\xi \xi, \quad \xi=\mathrm{e}^{-i f \tau \varphi},
\end{array}
$$


so that PCAC will fulfill.

By the canonical transformation $[1,5,6]$

$$
N=\mathbf{U}_{b}^{-b / 2} \psi, \quad \mathbf{U}_{b}^{b / 2}=\mathrm{e}^{i \gamma^{5} f \tau \varphi b}
$$

of the nucleon field, the original lagrangian $\mathcal{L}_{\psi \varphi}(5)$, that involves only the non-derivative pion-nucleon couplings, is transformed to the general form

$$
\mathcal{L}_{\psi \varphi} \rightarrow \mathcal{L}_{b N \varphi}=i \bar{N} \gamma^{\mu} \partial_{\mu} N+i \bar{N} \gamma^{\mu} \mathbf{U}_{b}^{-b / 2} \partial_{\mu} \mathbf{U}_{b}^{b / 2} N-\bar{N} \mathbf{U}_{b}^{b / 2} \mathbf{U} \mathbf{U}_{b}^{b / 2} N M,
$$

with $b$ being some dimensionless parameter. Then, with introducing the auxiliary quantities

$$
\begin{array}{r}
\xi^{b}=\mathrm{e}^{i b f \tau \varphi}, \\
V_{\mu}=\frac{i}{2}\left(\xi^{-b} \partial_{\mu} \xi^{b}+\xi^{b} \partial_{\mu} \xi^{-b}\right), \\
A_{\mu}=\frac{i}{2}\left(\xi^{-b} \partial_{\mu} \xi^{b}-\xi^{b} \partial_{\mu} \xi^{-b}\right),
\end{array}
$$

the total $S U(2) \times S U(2)$ invariant lagrangian is convenient to be generally rewritten as follows

$$
\begin{aligned}
& \mathcal{L}_{h}=\mathcal{L}_{N}+\mathcal{L}_{1 N \varphi}^{\text {int }}+\mathcal{L}_{0 N \varphi}^{\text {int }}+\mathcal{L}_{\varphi \varphi} \\
& \mathcal{L}_{N}=i \bar{N} \gamma^{\mu} \partial_{\mu} N, \\
& \mathcal{L}_{1 N \varphi}^{\text {int }}=\bar{N} \gamma^{\mu} V_{\mu} N+g^{\prime} \bar{N} \gamma^{\mu} A_{\mu} \gamma^{5} N= \\
& =\bar{N} \gamma^{\mu}\left\{-\tau\left[\varphi \times \partial_{\mu} \varphi\right] \frac{\sin ^{2}(z)}{\varphi^{2}}-g^{\prime} \gamma^{5}\left\{\frac{\tau \partial_{\mu} \varphi}{2 \sqrt{\varphi^{2}}} \sin (2 z)+\right.\right. \\
& \left.\left.+\frac{(\boldsymbol{\tau} \varphi)\left(\varphi \partial_{\mu} \varphi\right)}{\varphi^{2}} f b\left(1-\frac{\sin (2 z)}{2 z}\right)\right\}\right\} N \approx \\
& \approx-g^{\prime}(f b) \bar{N} \gamma^{\mu} \gamma^{5} \boldsymbol{\tau} \partial_{\mu} \varphi N-f^{2} b^{2} \bar{N} \gamma^{\mu}\left[\boldsymbol{\varphi} \times \partial_{\mu} \varphi\right] \tau N+\mathcal{O}\left(f^{3}\right), \quad z=f b \sqrt{\varphi^{2}}, \\
& \mathcal{L}_{0 N \varphi}^{\text {int }}=-\bar{N}\left[\exp \left(2 i \gamma^{5} f \boldsymbol{\tau} \varphi(b-1)\right)\right] N M \approx \\
& \approx-2 i f(b-1) M \bar{N} \gamma^{5} \tau \varphi N+2 f^{2}(b-1)^{2} \varphi^{2} \bar{N} N M-\bar{N} N M+\mathcal{O}\left(f^{2}\right), \\
& \mathcal{L}_{\varphi \varphi}=\frac{1}{2} \partial_{\mu} \varphi \partial^{\mu} \varphi\left(\frac{\sin ^{2}(y)}{y^{2}}\right)+\frac{\left(\varphi \partial_{\mu} \varphi\right)^{2}}{2 \varphi^{2}}\left(1-\frac{\sin ^{2}(y)}{y^{2}}\right) \approx \\
& \approx \frac{1}{2} \partial_{\mu} \varphi \partial^{\mu} \boldsymbol{\varphi}-\frac{2}{3} f^{2} \boldsymbol{\varphi}^{2}\left(\partial_{\mu} \varphi \partial^{\mu} \boldsymbol{\varphi}-\frac{\left(\varphi \partial_{\mu} \boldsymbol{\varphi}\right)^{2}}{\varphi^{2}}\right)+\mathcal{O}\left(f^{4}\right), \quad y=2 f \sqrt{\boldsymbol{\varphi}^{2}},
\end{aligned}
$$

where introducing a parameter $g^{\prime}$ does not spoil invariance of the lagrangian $[1,5-7]$. With adding the term $\mathcal{L}_{S B}$ (13), the lagrangian $\mathcal{L}_{\varphi \varphi}(23)$ transforms to

$$
\mathcal{L}_{\varphi \varphi S B}=\mathcal{L}_{\varphi \varphi}+\mathcal{L}_{S B} \approx \mathcal{L}_{\varphi \varphi}-\frac{m^{2}}{2} \varphi^{2}+\frac{m^{2}}{6} f^{2}(\varphi \varphi)^{2}+\mathcal{O}\left(f^{4}\right) .
$$

As seen, the lagrangian (19) incorporates both derivative and non-derivative pion-nucleon couplings. The relation among these two kinds of couplings is dictated by choice of $b$ value. Generally speaking, the parameter $b$ could be considered as a fit-parameter to be fixed by processing the appropriate experimental 
data. Chosen hereafter $b=1$, we arrive at the most frequently considered lagrangian comprising only the derivative pion-nucleon couplings, with $\mathcal{L}_{0 N \varphi}^{i n t}$ therein reduces merely to $-\bar{N} N M$. The effective lagrangian (19) with $b=1$ is known to be obtained in the general geometrical method [7-9], and also deduced starting with the $\sigma$-model when the $\sigma$-meson is finely eliminated $[1,10]$. At $b=0$ we would apparently return to the non-derivative couplings of pions and nucleons (5), (6), (12). In processing various experimental data, the various suggestions are managed for purpose of best fitting. For instance, the succeeding canonical transformation of the nucleon field, with the parameter $g^{\prime}$,

$$
N_{1} \rightarrow \mathrm{e}^{i g^{\prime} f \gamma^{5} \tau \varphi} N
$$

was performed in the work [11] so as the effective pion-nucleon lagrangian to work with would reduce to

$$
\begin{aligned}
\mathcal{L}_{N_{1}}+\mathcal{L}_{1 N_{1} \varphi}^{i n t}+ & \mathcal{L}_{0 N_{1} \varphi}^{i n t} \approx-i M g^{\prime} 2 f \bar{N}_{1} \gamma^{5} \boldsymbol{\tau} \varphi N_{1}+M g^{\prime 2} 2 f^{2} \varphi^{2} \bar{N}_{1} N_{1}+ \\
& +\left(g^{\prime 2}-1\right) f^{2} \bar{N}_{1} \gamma^{\mu}\left[\varphi \times \partial_{\mu} \varphi\right] \varphi N_{1}-\bar{N}_{1} N_{1} M+\mathcal{O}\left(f^{3}\right)
\end{aligned}
$$

Let us now treat the pion mass and wavefunction renormalization. The lagrangian (24) is expedient to be presented as

$$
\begin{array}{r}
\overline{\mathcal{L}}_{\varphi \varphi S B}=\mathcal{L}_{\varphi \varphi}^{0}+\overline{\mathcal{L}}_{\varphi \varphi}^{i n t}, \\
\mathcal{L}_{\varphi \varphi}^{0}=\frac{1}{2} \partial_{\mu} \varphi(x) \partial^{\mu} \boldsymbol{\varphi}(x)-\frac{m^{2}}{2} \varphi^{2}(x), \\
\overline{\mathcal{L}}_{\varphi \varphi}^{i n t}=\mathcal{M} \varphi^{2}(x)+\mathcal{K} \partial_{\mu} \varphi(x) \partial^{\mu} \varphi(x),
\end{array}
$$

with introducing the subsidiary quantities $\mathcal{K}, \mathcal{M}$. This lagrangian transforms immediately to the canonical form as follows

$$
\begin{array}{r}
\overline{\mathcal{L}}_{\varphi \varphi S B}=\frac{1}{2} \partial_{\mu} \boldsymbol{\varphi}(x) \partial^{\mu} \boldsymbol{\varphi}(x)[1+2 \mathcal{K}]-\frac{m^{2}}{2} \boldsymbol{\varphi}^{2}(x)\left[1-\frac{2 \mathcal{M}}{m^{2}}\right]= \\
=\frac{1}{2} \partial_{\mu} \boldsymbol{\varphi}_{r}(x) \partial^{\mu} \boldsymbol{\varphi}_{r}(x)-\frac{m^{2}}{2} \boldsymbol{\varphi}_{r}^{2}(x) \mathrm{z}\left[1-\frac{2 \mathcal{M}}{m^{2}}\right]= \\
=\frac{1}{2} \partial_{\mu} \boldsymbol{\varphi}_{r}(x) \partial^{\mu} \boldsymbol{\varphi}_{r}(x)-\frac{m_{r}^{2}}{2} \boldsymbol{\varphi}_{r}^{2}(x),
\end{array}
$$

where the renormalized wavefunction $\varphi_{r}$ and mass $m_{r}$ are identified as

$$
\boldsymbol{\varphi}_{r}(x)=\boldsymbol{\varphi}(x) \mathrm{z}^{-1 / 2}, \mathrm{z}^{-1}=1+2 \mathcal{K}, m_{r}^{2}=m^{2} \mathbf{z}\left(1-\frac{2 \mathcal{M}}{m^{2}}\right)=m^{2}\left(1-2 \mathcal{K}-\frac{2 \mathcal{M}}{m^{2}}\right) .
$$

By correlating the propagators

$$
\left\langle 0\left|\mathcal{T} \varphi_{\alpha}\left(x_{1}\right) \varphi_{\beta}\left(x_{2}\right) \exp \left[i \int \mathrm{d} x^{4} \hat{\mathcal{L}}_{\varphi \varphi}(x)\right]\right| 0\right\rangle,
$$

calculated with the interactions $\hat{\mathcal{L}}_{\varphi \varphi}=\mathcal{L}_{\varphi \varphi S B}-\mathcal{L}_{\varphi \varphi}^{0}(24)$ and $\hat{\mathcal{L}}_{\varphi \varphi}=\overline{\mathcal{L}}_{\varphi \varphi}^{i n t}(27)$, these quantities $\mathcal{M}$ and $\mathcal{K}$ are directly determined in terms of the vacuum expectations $\left\langle 0\left|\varphi^{2 m}(x)\right| 0\right\rangle$ and

$$
\delta_{a b} \Delta_{\mu \nu}=\left\langle 0\left|\partial_{\mu} \varphi_{a}(x) \partial_{\nu} \varphi_{b}(x)\right| 0\right\rangle=\delta_{a b} i \int \frac{\mathrm{d}^{4} k}{(2 \pi)^{4}} \frac{k_{\mu} k_{\nu}}{k^{2}-m^{2}+i \varepsilon},
$$


which eventually results in

$$
\begin{array}{r}
\mathcal{K}=\frac{2}{3} \sum_{n=1}^{\infty} \frac{(-1)^{n}(4 f)^{2 n}}{(2 n+2) !}\left\langle 0\left|\varphi^{2 n}(x)\right| 0\right\rangle=\frac{1}{3}\left\langle 0\left|\frac{\sin ^{2} y}{y^{2}}-1\right| 0\right\rangle, \quad y=2 f \sqrt{\boldsymbol{\varphi}^{2}(x)}, \\
2 \mathcal{M}=m^{2} \sum_{n=1}^{\infty} \frac{(-1)^{(n+1)}}{3}(2 f)^{2 n} \frac{2 n+3}{(2 n+1) !}\left\langle 0\left|\varphi^{2 n}(x)\right| 0\right\rangle+ \\
+\frac{1}{9}\left\langle 0\left|\partial_{\mu} \boldsymbol{\varphi}(x) \partial^{\mu} \boldsymbol{\varphi}(x)\right| 0\right\rangle \sum_{n=1}^{\infty}(-1)^{n}(4 f)^{2 n} \frac{2 n(2 n+1)}{(2 n+2) !}\left\langle 0\left|\varphi^{2(n-1)}(x)\right| 0\right\rangle= \\
=m^{2}\left\langle 0\left|1-\frac{1}{3}\left(\cos y+2 \frac{\sin y}{y}\right)\right| 0\right\rangle-\frac{2(4 f)^{2}}{9}\left\langle 0\left|\partial_{\mu} \varphi(x) \partial^{\mu} \boldsymbol{\varphi}(x)\right| 0\right\rangle \times \\
\times\left\langle 0\left|\left\{\frac{\sin ^{2} y}{2 y^{2}}+\frac{2}{(2 y)^{2}}\left(\frac{\sin 2 y}{2 y}-1\right)-\frac{1}{(2 y)^{2}}\left(\frac{\sin ^{2} y}{y^{2}}-1\right)\right\}\right| 0\right\rangle .
\end{array}
$$

To proceed, we utilize the directly obtained relations:

$$
\left\langle 0\left|\varphi^{2 n}(x)\right| 0\right\rangle=\Delta^{n} \cdot(2 n+1) ! !
$$

with

$$
\delta_{a b} \Delta=\left\langle 0\left|\varphi_{a}(x) \varphi_{b}(x)\right| 0\right\rangle=\delta_{a b} \int \frac{\mathrm{d}^{4} k}{(2 \pi)^{4}} \frac{i}{k^{2}-m^{2}+i \varepsilon},
$$

and then

$$
(2 n+1) ! !=\frac{2 \sqrt{\mathrm{p}}}{\sqrt{\pi}} \int_{0}^{\infty}\left(2 \mathrm{p} x^{2}\right)^{(n+1)} \exp \left[-\mathrm{p} x^{2}\right] \mathrm{d} x, \quad \mathrm{p}>0
$$

The integrals (35) and (31) diverge due to the singular high momenta behavior of the integrand. To interpret them and other divergent integrals hereafter, the dimensional regularization (or continues dimensional) method is relevant, see, for instance, refs. [1,12]. As ascertained in Appendix A, the quantities (35), (31) are presented in $d$-dimensions in the form

$$
\begin{aligned}
\Delta(d)=\frac{\mu^{d-4}}{(4 \pi)^{d / 2}} \frac{\left(m^{2}\right)^{d / 2-1}}{\Gamma(d / 2)} \pi(-1)^{n} \operatorname{cosec}[\pi d / 2], \quad d / 2=n+\nu_{d}, n=0, \pm 1, \pm 2, \ldots, \quad+0 \leq \operatorname{Re} \nu_{d} \leq 1-0 \\
\Delta_{\mu \nu}(d)=g_{\mu \nu} \frac{m^{2}}{d} \Delta(d)
\end{aligned}
$$

where, as usual, $\mu$ is an auxiliary quantity having dimension of mass, $\Gamma$ is the Euler Gamma-function, and physical limit corresponds to $d \rightarrow 4, \Delta(d) \rightarrow \Delta(4) \rightarrow+\infty$.

With the regularization (37), (38), the sequence of summation and integration can be interchanged in calculating the quantities $\mathcal{K}(32)$ and $\mathcal{M}(33)$ with the help of eq. (36). Then with allowance for eqs. $(31)$ - (36) we arrive at the eventual results for the quantities $\mathcal{K}, \mathcal{M}$, which determine the pion mass and field renormalization (29):

$$
\begin{array}{r}
\mathcal{K}=\frac{1}{3}(P(1-\exp [-1 / P])-1), \\
\mathcal{M}=\frac{m^{2}}{3}(\exp [-1 / P](1+P)-P)+\frac{m^{2}}{2}\left(1-\exp [-1 / 4 P]+\frac{1}{6 P} \exp [-1 / 4 P]\right),
\end{array}
$$


where

$$
P^{-1}=2 \Delta(2 f)^{2}
$$

The way we have been following here is akin to the well known methods of divergent series summation, see ref. [13].

The main feature to stress is that these quantities $\mathcal{K}, \mathcal{M}, z, m_{r}^{2}$ do not diverge, when $d \rightarrow 4$ and consequently $\Delta \rightarrow \infty, P \rightarrow 0$ (see Appendix A). Indeed, in this case we have got

$$
\mathcal{K}=-\frac{1}{3}, \frac{2 \mathcal{M}}{m^{2}}=1
$$

Thus, starting with the essentially nonlinear lagrangian (23), (24), we encounter no divergence in treating the mass and wavefunction renormalization. Let us mention that if we had restricted ourselves by the lowest $\varphi^{2}$ order, we should apparently have got at $d \rightarrow 4$ the divergent quantities

$$
\begin{array}{r}
\mathcal{K}_{1}=-(2 f)^{2} \frac{\Delta}{3}, \mathrm{z}_{1}^{-1}=1-\frac{2}{3} \Delta(2 f)^{2}, \quad \frac{2 \mathcal{M}_{1}}{m^{2}}=\frac{\Delta}{6}(2 f), \\
m_{r 1}^{2}=m^{2}\left(1+\frac{\Delta}{2}(2 f)^{2}\right),
\end{array}
$$

as discussed in the manifold investigations, see, for instance, ref. [1]. The method how to deal with this divergence is being elaborated within the chiral perturbation theory $[1,14]$.

The principle feature to stress is that the whole lagrangian

$$
\mathcal{L}_{S B h}=\mathcal{L}_{h}+\mathcal{L}_{S B}
$$

at any values of the parameters involved, is invariant under the global $S U(2) \times S U(2)$ isospin as well as chiral rotation, save for the symmetry-breaking term (13). Apparently, due to eqs. (14), (15), any transformation of the field $\varphi$ entails a corresponding transformation of the nucleon field $N$. The lagrangian (19) proves to be relevant in treating the low-energy electro-weak processes with non-strange hadrons, which is our purpose.

\section{Hadron currents}

The infinitesimal global gauge transformations

$$
\begin{array}{r}
\delta_{\varepsilon} \psi=-\frac{i}{2} \boldsymbol{\tau} \varepsilon \psi, \quad \delta_{\varepsilon} \mathbf{U}=-\frac{i}{2}[\boldsymbol{\tau} \varepsilon, \mathbf{U}], \quad \delta_{\varepsilon} \varphi_{s}=-\epsilon_{s t r} \varphi_{t} \varepsilon_{r}, \\
\delta_{a} \psi=\frac{i}{2} \gamma^{5} \boldsymbol{a} \boldsymbol{\tau} \psi, \quad \delta_{a} \mathbf{U}=-\frac{i}{2}\left\{\gamma^{5} \boldsymbol{a} \boldsymbol{\tau}, \mathbf{U}\right\}
\end{array}
$$

of the lagrangian $\mathcal{L}_{S B h}(44)$ yield the iso-triplet of hadronic vector $\mathcal{J}_{\mu}(x)$ and axial $\mathcal{J}_{5 \mu}(x)$ currents, $\left\{\mathcal{J}_{(5) \mu}^{r}(x)\right\}(r=$ $1,2,3)$, one current $\mathcal{J}_{(5) \mu}^{r}(x)$ of the iso-triplet for each $\varepsilon_{r}, a_{r}$ in eqs. (45), (46),

$$
\begin{gathered}
\mathcal{J}_{\mu}=\mathcal{J}_{N \mu}+\mathcal{J}_{\varphi \mu}, \\
\mathcal{J}_{5 \mu}=\mathcal{J}_{N 5 \mu}+\mathcal{J}_{\varphi 5 \mu},
\end{gathered}
$$


where

$$
\begin{array}{r}
\mathcal{J}_{N \mu}=\frac{1}{4} \bar{N} \gamma^{\mu}\left[\xi^{-b} \boldsymbol{\tau} \xi^{b}+\xi^{b} \boldsymbol{\tau} \xi^{-b}\right] N+\frac{g^{\prime}}{4} \bar{N} \gamma^{\mu} \gamma^{5}\left[\xi^{-b} \boldsymbol{\tau} \xi^{b}-\xi^{b} \boldsymbol{\tau} \xi^{-b}\right] N= \\
=\frac{1}{2} \bar{N} \gamma^{\mu} \boldsymbol{\tau} N+\bar{N} \gamma^{\mu}\left(\boldsymbol{\varphi}(\boldsymbol{\tau} \boldsymbol{\varphi})-\boldsymbol{\tau}(\boldsymbol{\varphi})^{2}\right) \frac{\sin ^{2} z}{\boldsymbol{\varphi}^{2}} N+ \\
+g^{\prime} \bar{N} \gamma^{\mu} \gamma^{5}[\boldsymbol{\tau} \times \boldsymbol{\varphi}] \frac{\sin 2 z}{2 \sqrt{\boldsymbol{\varphi}^{2}}} N \approx \bar{N} \gamma^{\mu} \frac{\boldsymbol{\tau}}{2} N+\mathcal{O}\left(f^{2}\right) \\
\mathcal{J}_{N 5 \mu}=\frac{g^{\prime}}{4} \bar{N} \gamma^{\mu} \gamma^{5}\left[\xi^{-b} \boldsymbol{\tau} \xi^{b}+\xi^{b} \boldsymbol{\tau} \xi^{-b}\right] N+\frac{1}{4} \bar{N} \gamma^{\mu}\left[\xi^{-b} \boldsymbol{\tau} \xi^{b}-\xi^{b} \boldsymbol{\tau} \xi^{-b}\right] N= \\
=\frac{g^{\prime}}{2} \bar{N} \gamma^{\mu} \gamma^{5} \boldsymbol{\tau} N+g^{\prime} \bar{N} \gamma^{\mu} \gamma^{5}\left(\boldsymbol{\varphi}(\boldsymbol{\tau} \boldsymbol{\varphi})-\boldsymbol{\tau}(\boldsymbol{\varphi})^{2}\right) \frac{\sin ^{2} z}{\boldsymbol{\varphi}^{2}} N+ \\
+\bar{N} \gamma^{\mu}[\boldsymbol{\tau} \times \boldsymbol{\varphi}] \frac{\sin 2 z}{2 \sqrt{\boldsymbol{\varphi}^{2}}} N \approx g^{\prime} \bar{N} \gamma^{\mu} \gamma^{5} \frac{\boldsymbol{\tau}}{2} N+\mathcal{O}\left(f^{2}\right), \\
\mathcal{J} \varphi \mu=-\frac{i}{16 f^{2}} \operatorname{Sp}\left[\boldsymbol{\tau}\left(\mathbf{U}_{\xi}^{\dagger} \partial_{\mu} \mathbf{U}_{\xi}+\mathbf{U}_{\xi} \partial_{\mu} \mathbf{U}_{\xi}^{\dagger}\right)\right]= \\
=\left(\frac{\sin y}{y}\right)^{2}\left[\boldsymbol{\varphi} \times \partial_{\mu} \boldsymbol{\varphi}\right] \approx\left[\boldsymbol{\varphi} \times \partial_{\mu} \boldsymbol{\varphi}\right]+\mathcal{O}\left(f^{3}\right) \\
\mathcal{J}_{\varphi 5 \mu}=\frac{-i}{16 f^{2}} \operatorname{Sp}\left[\boldsymbol{\tau}\left(\mathbf{U}^{\dagger} \partial_{\mu} \mathbf{U}-\mathbf{U} \partial_{\mu} \mathbf{U}^{\dagger}\right) \gamma^{5}\right]=\frac{-i}{16 f^{2}} \operatorname{Sp}\left[\boldsymbol{\tau}\left(\mathbf{U}_{\xi}^{\dagger} \partial_{\mu} \mathbf{U}_{\xi}-\mathbf{U}_{\xi} \partial_{\mu} \mathbf{U}_{\xi}^{\dagger}\right)\right]= \\
=\frac{\partial_{\mu} \boldsymbol{\varphi}}{2 f}\left(\frac{\sin 2 y}{2 y}\right)+f \frac{2 \varphi \partial_{\mu} \boldsymbol{\varphi}^{2}}{y^{2}}\left(1-\frac{\sin 2 y}{2 y}\right) \approx \frac{1}{2 f} \partial_{\mu} \boldsymbol{\varphi}+\mathcal{O}(f)
\end{array}
$$

The vector current is conserved,

$$
\partial^{\mu} \mathcal{J}_{\mu}=0
$$

Yet as the total lagrangian $\mathcal{L}_{S B h}(44)$ does besides $\mathcal{L}_{h}$ include the symmetry-breaking term $\mathcal{L}_{S B}$, the relation holds

$$
\partial^{\mu} \mathcal{J}_{5 \mu}=\frac{i m^{2}}{32 f^{2}} \operatorname{Sp}\left\{\left(\mathbf{U}_{\xi}^{\dagger}-\mathbf{U}_{\xi}\right), \boldsymbol{\tau}\right\}=\varphi \frac{m^{2}}{2 f} \frac{\sin y}{y} \approx \frac{m^{2}}{2 f} \boldsymbol{\varphi}+\mathcal{O}(f)
$$

the axial current is partially-conserved. It is to stress that the eqs. (53), (54) hold true just for the total currents, yet not for the nucleon (49), (50) and the pion (51), (52) currents themselves separately, so far the nucleon-pion interactions are involved into $\mathcal{L}_{h}$.

The compensating field $\varphi(x)$ is associated with the physical pion field,

$$
\pi^{ \pm}(x)=\frac{1}{\sqrt{2}}\left[\varphi_{1}(x) \mp i \varphi_{2}(x)\right], \quad \pi^{0}(x)=\varphi_{3}(x) .
$$

The lagrangian $\mathcal{L}_{S B h}(44)$ and the currents (47), (48) are expressed in terms of the iso-doublet of nucleons, the neutron and proton, $N=n, p$, and the iso-triplet of pions, $\pi^{r}, r= \pm, 0$.

The lagrangian $\mathcal{L}_{S B h}(x)$ (44) of considered system is also invariant under the global $U_{Y}(1)$ transformation, with the hypercharge $Y=1$ for the nucleon iso-doublet and $Y=0$ for the pion iso-triplet. Then invariance of $\mathcal{L}_{S B h}(x)$ under the $U_{Y}(1)$ transformation of nucleon fields,

$$
\psi_{N}^{\prime}(x) \Longrightarrow \psi_{N}(x)+\delta_{0} \psi(x), \quad \delta_{0} \psi(x)=-i \varepsilon_{0} \mathbf{I}_{N N^{\prime}} \psi_{N^{\prime}}(x) \frac{Y}{2}, \quad Y=1,
$$


yields the conserved neutral iso-scalar current

$$
\mathcal{J}_{\mu}^{0}(x)=\frac{1}{2} \mathbf{I}_{N N^{\prime}}\left(\bar{N} \gamma^{\mu} N^{\prime}\right)
$$

Certainly, any linear combination of the conserved (or partially-conserved) currents (47)-(52), (57) is a conserved (or partially-conserved) current as well.

The combination

$$
J_{\mu}^{0}(x) \equiv J_{\mu}^{e m}(x)=\mathrm{Z}_{e} \mathcal{J}_{\mu}^{3}(x)+\mathcal{J}_{\mu}^{0}(x)
$$

of the third component of the iso-vector current (47) and the iso-scalar current (57), is naturally understood to be the electromagnetic current, which stands to describe interaction of a hadronic system with an electromagnetic field. So, hereafter any current with the upper index 0 is implied to be the electromagnetic current, $J_{\mu}^{0}(x) \equiv J_{\mu}^{e m}(x)$.

The expectation value of electromagnetic current in the state $|N(p), 0\rangle$ containing no pions (pion vacuum) and a single nucleon, the proton or neutron, with a momentum $p$ is obtained (with the proper wave functions normalization) from eqs. (49), (57)

$$
\begin{array}{r}
\int\left\langle N, 0\left|J_{\mu}^{e m}(x)\right| N, 0\right\rangle \mathrm{d} \mathbf{x}= \\
=\chi_{N}^{*} \frac{p_{\mu}}{p_{0}}\left[\frac{1}{2}\left(\mathbf{Z}_{e} \tau_{0}+\mathbf{I}\right)-\mathrm{Z}_{e} \tau_{0} \frac{2}{3}\left\langle 0\left|\sin ^{2} z\right| 0\right\rangle\right] \chi_{N}, \quad\left(z=f \sqrt{\varphi^{2}(x)}\right)
\end{array}
$$

where $\chi_{N}$ stands for nucleon isospinor. To this expression at $\mu=0$ reduce to zero for the neutron $(N=n)$ and to unite for the proton $(N=p)$, as required, there should be

$$
\mathrm{Z}_{e}=\left(1-\frac{4}{3}\left\langle 0\left|\sin ^{2} z\right| 0\right\rangle\right)^{-1}
$$

Here the vacuum expectation is evaluated just likewise the akin quantities in the expressions (32)-(36), so that we have got

$$
\left\langle 0\left|\sin ^{2} z\right| 0\right\rangle=\frac{1}{2}-\frac{2 P-1}{4 P} \exp \left[-\frac{1}{4 P}\right]
$$

with $P$ given by eq. (41). Of course, in the lowest $\varphi^{2}$-order there would be $Z_{e}=1$. At the physical limit, $d \rightarrow 4, \Delta \rightarrow \infty$ in eqs. (37), (38) (see Appendix A), this reduces to

$$
\left\langle 0\left|\sin ^{2} z\right| 0\right\rangle=\frac{1}{2}
$$

and we have got $Z_{e}=3$, in treating the essentially non-linear current (49).

In the usual way, the first and second components of isovector current $\mathcal{J}_{\mu}^{r=1,2}(x)$ serve to construct the charge vector currents

$$
J_{N \mu}^{ \pm}(x)=\mathrm{Z}_{e}\left(\mathcal{J}_{N \mu}^{1}(x) \pm i \mathcal{J}_{N \mu}^{2}(x)\right)
$$


with the same parameter $Z_{e}$ as in eq. (58) where it occurred as a factor by the third component of isovector current. As one observes, the current $J_{(5) \mu}^{+}(x)$ would increase whereas the current $J_{(5) \mu}^{-}(x)$ decrease electric charge of a hadron system by unity. The currents $J_{(5) \mu}^{ \pm}(x)$ occur in treating the $\beta$-decay of hadrons. For instance, the vector part of $n \rightarrow p$ weak transition current is the matrix element of $J_{N \mu}^{+}(x)$ (63) between single neutron and single proton states (and pion vacuum)

$$
\left\langle p, 0\left|J_{N \mu}^{+}\right| n, 0\right\rangle=\mathrm{Z}_{e} \bar{U}_{p} \gamma_{\mu}\left(1-\frac{4}{3}\left\langle 0\left|\sin ^{2} z(x)\right| 0\right\rangle\right) U_{n},
$$

where $\bar{U}_{p}, U_{n}$ are the proton and neutron wave amplitudes. Thereby one infers the parameter $Z_{e}$ emerges again to be defined by the expression (60).

Calculating the axial part of the respective transition current serves to express the parameter $g^{\prime}$ in the lagrangian (21) through the nucleon axial form-factor $g_{A}$ :

$$
\begin{array}{r}
\left\langle p, 0\left|J_{N 5 \mu}^{+}(x)\right| n, 0\right\rangle=\left\langle p, 0\left|\mathcal{J}_{N 5 \mu}^{1}(x)+i \mathcal{J}_{N 5 \mu}^{2}(x)\right| n, 0\right\rangle= \\
=g^{\prime} \bar{U}_{p} \gamma_{\mu} \gamma_{5}\left(1-\frac{4}{3}\left\langle 0\left|\sin ^{2} z(x)\right| 0\right\rangle U_{n}=g_{A} \bar{U}_{p} \gamma_{\mu} \gamma^{5} U_{n}\right.
\end{array}
$$

with the vacuum expectation value given by eq. (61). Thus we have got

$$
g^{\prime}=g_{A}\left\{1-\frac{1}{3}\left(2-\frac{2 P-1}{P} \exp [-1 / 4 P]\right)\right\}^{-1}
$$

which reduces to

$$
g^{\prime}=3 g_{A}
$$

for the physical limit, $d \rightarrow 4, \Delta \rightarrow \infty, P \rightarrow 0$ (see Appendix A), of eqs. (37), (41). Of course, in the lowest $\varphi^{2}$-order $\left(P^{-1} \rightarrow 0\right)$ there would merely be $g^{\prime}=g_{A}$.

With having recourse to the Standard Model (SM) concepts (see, for instance, [1]), the third component $\mathcal{J}_{(5) \mu}^{3}(x)$ of the isotriplet current $\mathcal{J}_{(5) \mu}(x)$ and the iso-scalar current $\mathcal{J}_{\mu}^{0}$ are still combined giving, besides $J_{\mu}^{e m}(x)$, the neutral weak currents

$$
\begin{array}{r}
J_{\mu}^{Z}(x)=\mathrm{Z}_{e} \mathcal{J}_{\mu}^{3}(x)\left(1-2 s_{W}^{2}\right)-2 \mathcal{J}_{\mu}^{0}(x) s_{W}^{2}, \quad s_{W}^{2}=1-\frac{M_{W}^{2}}{M_{Z}^{2}}, \\
J_{5 \mu}^{Z}(x)=\mathcal{J}_{5 \mu}^{3}
\end{array}
$$

Here the coefficients are chosen so that these currents (68), (69) would serve to describe the feasible hadron transitions in the neutral $Z^{0}$-boson field (see, for instance, [1]).

\section{The hadron interaction with gauge fields.}

The hadron currents have been considered, we are now to describe the hadron (pion and nucleon) interactions with the electromagnetic field $\mathcal{A}_{\mu}^{e m}(x)$, and with the charged $\mathcal{A}_{\mu}^{ \pm}(x)$ and neutral $\mathcal{A}_{\mu}^{Z}(x)$ fields associated with the $W^{ \pm}$- and $Z^{0}$-boson fields. The electro-weak lagrangian $\mathcal{L}_{\text {int }}^{E W}(x)$ to describe these 
interactions originates from requirement of $\mathcal{L}_{h}(19)$ invariance under the local (i.e. with the space-time dependent parameters $\varepsilon(x), \mathbf{a}(x)$ ) gauge transformation (45), (46), (56).

In order for the local transformation (45), (46), (56) to be an invariance of the lagrangian $\mathcal{L}_{h}(x)$ (19), the derivatives $\partial_{\mu} \psi_{N}(x), \partial_{\mu} \mathbf{U}(x)$ in $\mathcal{L}_{h}(x)$ are known (see, for instance, [1, 15-17]) to be replaced by the respective covariant derivatives $\mathcal{D}_{\mu}(x) \psi_{N}(x), \mathcal{D}_{\mu}(x) \mathbf{U}(x)$, which read for our consideration as

$$
\partial_{\mu} \psi_{N}(x) \Longrightarrow \mathcal{D}_{\mu}(x) \psi_{N}(x)=\partial_{\mu} \psi_{N}(x)+i e \mathcal{A}_{\mu}^{a}(x) \ell_{N N^{\prime}}^{a} \psi_{N^{\prime}}(x), \quad e=\sqrt{4 \pi \alpha},
$$

where

$$
\begin{array}{r}
\ell_{N N^{\prime}}^{a}=\frac{1}{2} \delta_{a 0}\left(\mathbf{I}_{N N^{\prime}}+\mathbf{Z}_{e} \tau_{N N^{\prime}}^{0}\right)+a^{2} \tau_{N N^{\prime}}^{a}\left(\mathbf{Z}_{e}-\gamma^{5}\right), \quad a=0, \pm, \\
\tau^{0}=\tau_{3}, \quad \tau^{ \pm}=\left(\tau_{1} \pm i \tau_{2}\right) / 2, \\
\ell_{N N^{\prime}}^{Z}=-\mathbf{I}_{N N^{\prime}} s_{W}^{2}+\frac{1}{2} Z_{e} \tau_{N N^{\prime}}^{0}\left(1-2 s_{W}^{2}\right)-\gamma^{5} \frac{1}{2} \tau_{N N^{\prime}}^{0}, \quad a=Z,
\end{array}
$$

and

$$
\begin{array}{r}
\partial_{\mu} \mathbf{U}(x) \Longrightarrow \mathcal{D}_{\mu}(x) \mathbf{U}(x)=\partial_{\mu} \mathbf{U}(x)+\frac{i}{2} e \mathcal{A}_{\mu}^{0}(x)\left[\tau^{0}, \mathbf{U}(x)\right]+ \\
+i e \mathcal{A}_{\mu}^{ \pm}(x)\left(\left[\tau^{ \pm}, \mathbf{U}(x)\right]+\left\{\tau^{ \pm} \gamma^{5}, \mathbf{U}(x)\right\}\right)+ \\
+\frac{i e}{2} \mathcal{A}_{\mu}^{Z}(x)\left(\left[\tau^{0}, \mathbf{U}(x)\right]\left(1-2 s_{W}^{2}\right)+\left\{\tau^{0} \gamma^{5}, \mathbf{U}(x)\right\}\right) .
\end{array}
$$

The parameter $Z_{e}$ (60) involved herein has been determined so that to provide the proper description of hadron neutral and charge currents. Then the electroweak lagrangian $\mathcal{L}_{\text {int }}^{E W}(x)$ results in terms of the hadron currents $J_{\mu}^{a}(x)$ (47)-(69) and the fields $\mathcal{A}_{\mu}^{a}(x)$, which are related to the physical fields $A_{\mu}^{e m}(x), W_{\mu}^{ \pm}(x), Z_{\mu}^{0}(x)$, and the effective coupling constants by the equations

$$
\begin{aligned}
\mathcal{A}_{\mu}^{0}(x)=A_{\mu}^{e m}(x), e \mathcal{A}_{\mu}^{ \pm}(x)=\frac{\left|V_{u d}\right| \sqrt{G} M_{W}}{2^{1 / 4}} W_{\mu}^{ \pm}, e \mathcal{A}_{\mu}^{Z}(x) & =\frac{\left|V_{u d}\right| \sqrt{2 G} M_{Z}}{2^{1 / 4}} Z_{\mu}^{0}, \\
\frac{\sqrt{G}}{2^{1 / 4}} & =\frac{|e|}{2 \sqrt{2} s_{W} M_{W}},
\end{aligned}
$$

with the Fermi interaction constant $G$, the $W^{ \pm}$- and $Z^{0}$ - boson masses $M_{W}, M_{Z}$, and the $C K M$-quarkmixing matrix element $\left|V_{u d}\right|$ [18], so that to provide true description of hadron interactions with the electromagnetic and heavy gauge boson fields [1, 19].

Then we arrive at the effective lagrangian to explore the pion and nucleon interactions with the gauge fields,

$$
\begin{array}{r}
\mathcal{L}_{\text {int }}^{E W}(x)=L^{e m}(x)+L^{1 W}(x)+L^{W e m}(x)+L^{1 Z}(x)+L^{Z e m}(x), \\
L^{e m}(x)=L^{1 e m}(x)+L^{2 e m}(x), \\
L^{1 e m}(x)=-e \mathcal{A}_{\mu}^{0}(x) J^{0 \mu}(x)=-e A_{\mu}^{e m}(x) J^{e m \mu}(x), \\
L^{2 e m}(x)=e^{2} \mathcal{A}_{\mu}^{0} \mathcal{A}^{0 \mu} \pi^{-}(x) \pi^{+}(x) \frac{\sin ^{2} y}{y^{2}}=e^{2} A_{\mu}^{e m} A^{e m \mu} \frac{\sin ^{2} y}{y^{2}} \pi^{-}(x) \pi^{+}(x),
\end{array}
$$




$$
\begin{array}{r}
L^{1 W}(x)=-e \mathcal{A}^{a \mu}(x) \tilde{J}_{\mu}^{a}(x)=-\frac{\left|V_{u d}\right| \sqrt{G} M_{W}}{2^{1 / 4}} \tilde{J}_{\mu}^{a}(x) W^{a \mu}(x), \quad a= \pm, \\
L^{W e m}(x)=-e A_{\mu}^{e m}(x) \frac{\left|V_{u d}\right| \sqrt{G} M_{W}}{2^{1 / 4}} W^{a \mu}(x) \sqrt{2} \pi^{-a}\left(\pi^{0} \frac{\sin ^{2}(y)}{y^{2}}+a \frac{i}{2 f} \frac{\sin (2 y)}{2 y}\right), \\
L^{1 Z}(x)=-\frac{\left|V_{u d}\right| \sqrt{2 G} M_{Z}}{2^{1 / 4}} \tilde{J}_{\mu}^{Z}(x) Z^{0 \mu}(x), \\
L^{Z e m}(x)=e A_{\mu}^{e m}(x) \frac{\left|V_{u d}\right| \sqrt{2 G} M_{Z}}{2^{1 / 4}} Z^{0 \mu}(x) \frac{\sin ^{2}(y)}{y^{2}}\left(1-2 s_{W}^{2}\right) \pi^{+}(x) \pi^{-}(x) .
\end{array}
$$

All the interactions quadratic in the fields $\mathcal{A}_{\mu}^{ \pm}(x), \mathcal{A}_{\mu}^{Z}(x)$ are abandoned in $\mathcal{L}^{E W}(x)(75)$, yet the interactions $L^{2 e m}(78), L^{W e m}(80), L^{Z e m}(82)$, incorporating the products of fields, $A_{\mu}^{e m} A^{e m \mu}, A_{\mu}^{e m} W^{a e m}, A_{\mu}^{e m} Z^{0 \mu}$, are presented therein. Thus, the lagrangian $\mathcal{L}_{\text {int }}^{E W}$ enables one to describe the electro-weak transitions accompanied immediately by $\gamma$-radiation. The expressions $(77),(79),(81)$ are written in terms of the currents

$$
J_{\mu}^{0}(x) \equiv J_{\mu}^{e m}(x), \quad \tilde{J}_{\mu}^{ \pm}(x)=J_{\mu}^{ \pm}(x)+J_{5 \mu}^{ \pm}, \quad \tilde{J}_{\mu}^{Z}(x)=J_{\mu}^{Z}(x)+J_{5 \mu}^{Z}
$$

where the currents $J_{(5) \mu}^{0, \pm, Z}$ are derived in Sec. III. As realized, the emission (absorption) of $W^{ \pm}-, Z^{0}$-bosons by hadrons is described by the lagrangian (75).

The total lagrangian $\tilde{\mathcal{L}}(x)$ to describe the hadron electro-weak transitions in considered system is the sum of the lagrangians $\mathcal{L}_{S B h}(x)(44)$ and $\mathcal{L}_{\text {int }}^{E W}(x)(75)$,

$$
\tilde{\mathcal{L}}(x)=\mathcal{L}_{S B h}(x)+\mathcal{L}_{\text {int }}^{E W}(x) .
$$

The lagrangian (75), (84) is to be complemented by the ordinary lagrangian $\ell^{E W}(x)$ to describe the interactions of leptons with photons and heavy gauge bosons :

$$
\begin{array}{r}
\ell^{E W}(x)=\ell^{e m}(x)+\ell^{1 W}(x)+\ell^{1 Z}(x), \\
\ell^{e m}(x)=-e \bar{\psi}_{l}(x) \gamma^{a} \psi_{l}(x) A_{a}^{e m}(x), \quad l=e, \mu, \tau, \\
\ell^{1 W}(x)=-\frac{M_{W} \sqrt{G}}{2^{1 / 4}}\left(W_{a}^{+}(x) \bar{\psi}_{\nu}(x) \gamma^{a}\left(1+\gamma^{5}\right) \psi_{l}(x)+\right. \\
\left.+W_{a}^{-}(x) \bar{\psi}_{l}(x) \gamma^{a}\left(1+\gamma^{5}\right) \psi_{\nu}(x)\right), \\
\ell^{1 Z}(x)=-\frac{M_{Z} \sqrt{2 G}}{2^{1 / 4}} Z^{0}\left(\frac{1}{2} \bar{\psi}_{\nu}(x) \gamma^{a}\left(1+\gamma^{5}\right) \psi_{\nu}(x)+\right. \\
\left.+\bar{\psi}_{l}(x) \gamma^{a}\left(-\frac{1}{2}\left(1+\gamma^{5}\right)+2 s_{W}^{2}\right) \psi_{l}(x)\right),
\end{array}
$$

were $\psi_{\nu}, \psi_{l=e, \mu, \tau}$ stand for the neutrino, and electron, muon, $\tau$-lepton fields.

The contribution $\mathcal{L}_{\text {gauge }}\left(\mathcal{A}^{a}, \partial_{\mu} \mathcal{A}^{a}\right)$ to the total lagrangian from the interactions of the gauge fields $\mathcal{A}_{\mu}^{a}(x)$ themselves is not immediately engaged into consideration of the low-energy electro-weak transitions of hadrons. So, there is here no need to plunge into its construction and add $\mathcal{L}_{\text {gauge }}\left(\mathcal{A}^{a}, \partial_{\mu} \mathcal{A}^{a}\right)$ to the effective lagrangian of the considered system.

The lagrangians we have been treating incorporate a number of parameters which are specified by correlating the evaluated physical quantities with the appropriate experimental data. 
Physical content and magnitude of the primary parameter $f$ is acquired by considering the pion weak decay,

$$
\pi^{+} \Longrightarrow \mu^{+} \nu_{\mu}\left(e^{+} \nu_{e}\right)
$$

The interaction

$$
L^{\pi}(x)=\ell^{1 W}(x)+L^{1 W}(x)
$$

causing this process is read of from the expressions (79), (83), (87). With proper allowance for eqs. (52), (74), (34)-(37), the $S_{\pi}-$ matrix element to describe the transition (89) is given by

$$
\begin{array}{r}
\left\langle\nu, \mu^{+}\left|S_{\pi}\right| \pi^{+}\right\rangle=-\frac{1}{2}\left\langle\nu, \mu^{+}\left|\mathcal{T} \int \mathrm{d}^{4} x_{1} \int \mathrm{d}^{4} x_{2} L^{\pi}\left(x_{1}\right) L^{\pi}\left(x_{2}\right)\right| \pi^{+}\right\rangle= \\
=-\left\langle\nu, \mu^{+}\right| \mathcal{T} \int \mathrm{d}^{4} x_{1} \int \mathrm{d}^{4} x_{2} J_{\varphi 5 \alpha}^{-}\left(x_{1}\right) W^{-\alpha}\left(x_{1}\right) \times \\
\times \bar{\psi}_{\nu}\left(x_{2}\right) \gamma^{\beta}\left(1+\gamma^{5}\right) \psi_{\mu}\left(x_{2}\right) W_{\beta}^{+}\left(x_{2}\right)\left|\pi^{+}\right\rangle \frac{G\left|V_{u d}\right| M_{W}^{2}}{\sqrt{2}}= \\
=(2 \pi)^{4} \delta\left(P_{\pi}-p_{\nu}-p_{\mu}\right) \frac{G\left|V_{u d}\right|}{2 \tilde{f}} u_{\pi} P_{\pi}^{\alpha} \cdot\left(\bar{u}_{\nu} \gamma_{\alpha}\left(1+\gamma^{5}\right) u_{\mu}\right),
\end{array}
$$

where the renormalized quantity $\tilde{f}$, associated now with the pion decay (89) vertex, appears in place of the initial, non-renormalized parameter $f$ :

$$
\begin{array}{r}
\frac{1}{2 \tilde{f}}=\frac{1}{2 f}\left(1+\frac{1}{3} \sum_{n=1}^{\infty} \frac{(-1)^{n}(4 f)^{2 n}}{(2 n+1) !}\left\langle 0\left|\varphi^{2 n}\right| 0\right\rangle\right)=\frac{1}{2 f} \\
\left(1+\frac{1}{3}\left\langle 0\left|\frac{\sin 2 y}{2 y}-1\right| 0\right\rangle\right)= \\
=\frac{1}{2 f} \frac{1}{3}(2+\exp [-1 / P])
\end{array}
$$

with $P$ given by eq. (41). In the expression (91), $P_{\pi}, u_{\pi}, p_{\nu}, u_{\nu}, p_{\mu}, u_{\mu}$ stand for momenta and wave amplitudes of the pion, neutrino and muon respectively. Needless to say all these momenta are negligible as compared to the gauge boson mass $\mathrm{M}_{W}$, that appears in calculating the quantity (91) through the $W$-boson propagator. By way of illustration, the amplitude (91), as caused by the interaction (90), can be displayed by the diagram, fig. 1 . , where the small disk stands for $W$ coupling with leptons, and the double-circle figures pion- $W$ coupling, with all the infinite number of virtual pion propagators. The solid line depicts a decaying pion, and the zigzag one displays a $W$-boson propagator; the thin lines stand for final leptons. (As $M_{W} \rightarrow \infty$, the zigzag gets constricted in point, $x_{1} \rightarrow x_{2}$.)

The transition amplitude (91) is still modified by the pion wave function renormalization

$$
u_{\pi}=u_{\pi r} \mathbf{z}^{1 / 2}=u_{\pi r}(1-\mathcal{K}),
$$

according to Sec. 2, eq. (29). That results in the additional renormalization of the amplitude (91) by replacing

$$
\begin{array}{r}
\frac{1}{2 \tilde{f}} \Longrightarrow \frac{1}{2 f_{r}}=\frac{1}{2 \tilde{f}}\left(1-\frac{1}{3}(P(1-\exp [-1 / P])-1)\right)= \\
=\frac{1}{2 f}\left\{1+\frac{1}{3}(\exp [-1 / P]-1)-\frac{1}{3}(P(1-\exp [-1 / P])-1)\right\},
\end{array}
$$


which is to be equated with the pion decay constant,

$$
\frac{1}{2 f_{r}}=F_{\pi} \approx 92.3 \mathrm{MeV}
$$

The point to emphasize is that no divergence occurs in calculating the renormalized decay amplitude (91), (94), just alike in calculating the quantities (29), (59), (64), albeit the vacuum expectation (37) itself diverges at the physical limit: $\Delta \rightarrow \infty, P \rightarrow 0$, when $d \rightarrow 4$ (see Appendix A). In this case one obtains from eq. (94) the plain relation

$$
\frac{1}{2 f}=\frac{1}{2 f_{r}}=F_{\pi}
$$

to express the parameter $f$, that resides in the effective lagrangian, through the phenomenological pion decay constant $F_{\pi}$.

It is expedient to correlate the parameters $g^{\prime}, g_{A}, f, F_{\pi}$ residing in the lagrangian (19) with the pionnucleon amplitude $g_{N N \pi}$ which would determine the simplest Lorenz-invariant pion-nucleon interaction,

$$
\mathcal{L}_{N N \pi}=i g_{N N \pi} \bar{N} \gamma_{5}(\boldsymbol{\tau} \varphi) N
$$

According to the interaction $\mathcal{L}_{1 N \varphi}^{i n t}(21)$, we obtain the amplitude of the charge (negative) pion emission process $n \rightarrow p+\pi^{-}$(the pion-nucleon vertex) as follows

$$
\begin{array}{r}
\left\langle p, \pi^{-}\left|S_{n p \pi}\right| n, 0\right\rangle=-i g^{\prime}\left\langle p, \pi^{-}\right| \int \mathrm{d}^{4} x \bar{N} \gamma^{\mu} \gamma^{5} \times \\
\times\left\{\frac{\boldsymbol{\tau} \partial_{\mu} \boldsymbol{\varphi}}{2 \sqrt{\boldsymbol{\varphi}^{2}}} \sin 2 z+(\boldsymbol{\tau} \boldsymbol{\varphi})\left(\boldsymbol{\varphi} \partial_{\mu} \boldsymbol{\varphi}\right) f \frac{1}{\boldsymbol{\varphi}^{2}}\left(1-\frac{\sin 2 z)}{2 z}\right)\right\} N|n, 0\rangle= \\
=g^{\prime} f \sqrt{2}(2 \pi)^{4} \delta\left(p_{n}-p_{p}-q_{\pi}\right) \\
\left\{\frac{1}{3}(1-\exp [-1 / 4 P])+\exp [-1 / 4 P]\right\} u_{\pi r} \bar{U}_{p} \gamma_{\mu} \gamma^{5} q_{\pi}^{\mu} U_{n},
\end{array}
$$

where $p_{n}, p_{p}, q_{\pi}$ are neutron, proton and pion (off-mass-shell) momenta, and we have evaluated the expectation values as we did thus far, $P$ being defined by eq. (41). The parameter $g^{\prime}$ is related to the nucleon axial form-factor $g_{A}$ by eqs. (66), (67), and $f$ is given in terms of the pion decay constant $F_{\pi}$ according to eqs. (94)-(96). In the physical limit, $d \rightarrow 4, P \rightarrow 0$ (see Appendix A), and $g^{\prime} \rightarrow 3 g_{A}$, this eq.(98) reduces to

$$
\left\langle p, \pi^{-}\left|S_{n p \pi}\right| n, 0\right\rangle=g_{A} f \sqrt{2}(2 \pi)^{4} \delta\left(p_{n}-p_{p}-q_{\pi}\right) u_{\pi r} \bar{U}_{p} \gamma_{\mu} \gamma^{5} q_{\pi}^{\mu} U_{n} .
$$

Using the Dirac equation for on-mass-shell nucleons, the expression (98) transforms to

$$
\begin{aligned}
\left\langle p, \pi^{-}\left|S_{n p \pi}\right|\right. & n, 0\rangle=-g^{\prime}\left(M_{n}+M_{p}\right) f \sqrt{2}(2 \pi)^{4} \delta\left(p_{n}-p_{p}-q_{\pi}\right) \times \\
& \times\left\{\frac{1}{3}(1-\exp [-1 / 4 P])+\exp [-1 / 4 P]\right\} u_{\pi r} \bar{U}_{p} \gamma^{5} U_{n} .
\end{aligned}
$$

On the other hand, with describing $N \pi$-interaction by eq. (97), the emission amplitude of a pion with the same wave amplitude $u_{\pi r}$ proves to be

$$
\left\langle p, \pi^{-}\left|S_{n p \pi}\right| n, 0\right\rangle=-g_{N N \pi}\left\langle p, \pi^{-}\left|\int \bar{N} \gamma^{5}(\boldsymbol{\tau} \varphi) N \mathrm{~d}^{4} x\right| n, 0\right\rangle=
$$




$$
=-g_{N N \pi} \sqrt{2}(2 \pi)^{4} \delta\left(p_{n}-p_{p}-q_{\pi}\right) u_{\pi r} \bar{U}_{p} \gamma^{5} U_{n}
$$

From the eqs. (101) and (100) one infers

$$
g_{N N \pi}=g^{\prime}\left(M_{p}+M_{n}\right) f\left\{\frac{1}{3}(1-\exp [-1 / 4 P])+\exp [-1 / 4 P]\right\} .
$$

At the physical limit, that is at $d \rightarrow 4, \Delta \rightarrow \infty, P \rightarrow 0$ (see Appendix A) in the expressions (37), (41) and $g^{\prime}=3 g_{A}$ according to eq. (67), this reduces to

$$
g_{N N \pi}=\frac{\left(M_{p}+M_{n}\right) g_{A}}{2 F_{\pi}}
$$

which is generally referred to as the Goldberger-Teiman identity [20]. So, in treating the essentially nonlinear pion-nucleon lagrangian, this well known relation is valid. Thus, all the parameters involved in our treatment have been specified.

\section{On the pion-nucleon weak interaction.}

The developed lagrangian $\mathcal{L}_{\text {int }}^{E W}(75)$ describes the hadron interaction with the gauge fields. This enables one to consider the weak hadron-hadron interaction, which is due to the $W^{ \pm}-, Z^{0}$-bosons exchange. The $P$-invariance violation in pion-nucleon interactions is a generally received manifestation of such processes [21-24]. The hadron weak-interaction physics is comprised in the parity-violating $N N \pi$-vertex. The charge (negative) pion emission $n \rightarrow p+\pi^{-}$, that was discussed in Sec. 4, typifies these phenomena. Side by side with the expression (98), the total amplitude of this reaction (the total vertex) comprises a part caused by the interaction $L^{1 W}$ (79) which violates $P$-parity,

$$
\begin{array}{r}
\left\langle p, \pi^{-}\left|S_{n p \pi}^{W}\right| n, 0\right\rangle=-\frac{1}{2}\left\langle p, \pi^{-}\left|\mathcal{T} \int \mathrm{d}^{4} x_{1} \int \mathrm{d}^{4} x_{2} L^{1 W}\left(x_{1}\right) L^{1 W}\left(x_{2}\right)\right| n, 0\right\rangle= \\
=-Q^{2}\left\langle p, \pi^{-}\right| \mathcal{T} \int \mathrm{d}^{4} x_{1} \int \mathrm{d}^{4} x_{2} \int \frac{\mathrm{d}^{4} P}{(2 \pi)^{4}} \times \\
\times \mathcal{D}_{\mu \nu}(P) \exp \left[-i P\left(x_{1}-x_{2}\right)\right] \tilde{J}_{\mu}^{+}\left(x_{1}\right) \tilde{J}_{\nu}^{-}\left(x_{2}\right)|n, 0\rangle, \\
Q=\frac{\left|V_{u d}\right| \sqrt{G} M_{W}}{2^{1 / 4}} .
\end{array}
$$

Here the $W$-boson propagator

$$
\mathcal{D}_{\mu \nu}(P)=\frac{-i g_{\mu \nu}}{P^{2}-M_{W}^{2}+i \epsilon}
$$

and the non-linear in $\varphi^{2}$ hadron currents are defined by eqs. (83), (63), (49)-(52). For illustration's sake (only), the amplitude (104) could be depicted by the diagram, fig. 2., where the zigzag line figures the pion interaction with nucleon via $W$-boson. The thin lines around zigzag are to simulate the infinite number of the virtual pion propagators (the virtual pions cloud), which appears in calculating the quantity (104), as the lagrangian (79) is of-principle non-linear in pion field. 
The amplitude given by eq. (104) could be said to be akin to the one given by eq. (91), in the sense that they both describe the processes caused by an intermediate $W$-boson.

The total amplitude of the treated pion emission (the total $N N \pi^{-}$-vertex) would be written as the sum of the amplitudes given by eqs. (98) and (104),

$$
\left\langle p, \pi^{-}\left|S_{n p \pi}^{t o t}\right| n, 0\right\rangle=\left\langle p, \pi^{-}\left|S_{n p \pi}\right| n, 0\right\rangle+\left\langle p, \pi^{-}\left|S_{n p \pi}^{W}\right| n, 0\right\rangle .
$$

In our approach, the $P$-odd, as well as $P$-conserving, transition amplitudes are directly dictated by the developed lagrangian (19), (75). So, there sees no reason to invent a phenomenological $P$-odd pion-nucleon interaction from general arguments.

The ensuing evaluations get simplified as all the momenta and masses involved are rather negligible as compared with the $W$-boson mass: $p_{n}, p_{p}, q_{\pi}, M_{n, p}, m \ll M_{W}$.

Performing the ordinary transformations we obtain

$$
\begin{array}{r}
\left\langle p, \pi^{-}\left|S_{n p \pi}^{W}\right| n, 0\right\rangle=i Q^{2} \int \mathrm{d}^{4} x_{1} \int \mathrm{d}^{4} x_{2} \int \frac{\mathrm{d}^{4} P}{(2 \pi)^{4}} \frac{\exp \left[-i P\left(x_{1}-x_{2}\right)\right]}{P^{2}-M_{W}^{2}+i \epsilon} \times \\
\times\left\{\bar{U}_{p} \gamma^{\mu}\left(\mathrm{Z}_{e}+g^{\prime} \gamma^{5}\right) U_{n} \exp \left[i x_{1}\left(p_{p}-p_{n}\right)\right] \times\right. \\
\times\left\langle\pi^{-}\right|\left(1+\left(\pi^{-}\left(x_{1}\right) \pi^{+}\left(x_{1}\right) \pi^{2}\left(x_{1}\right)\right) \frac{\sin ^{2} z\left(x_{1}\right)}{\pi^{2}\left(x_{1}\right)}\right)\left(\frac{\sqrt{2}}{2 f} \partial_{\mu} \pi^{+}\left(x_{2}\right) \frac{\sin 2 y\left(x_{2}\right)}{2 y\left(x_{2}\right)}+\right. \\
\left.+4 f \sqrt{2} \pi^{+}\left(x_{2}\right) \sum_{a= \pm, 0} \partial_{\mu} \pi^{a}\left(x_{2}\right) \pi^{-a}\left(x_{2}\right) \frac{1}{y^{2}\left(x_{2}\right)}\left(1-\frac{\sin 2 y\left(x_{2}\right)}{2 y\left(x_{2}\right)}\right)\right)|0\rangle+ \\
\left.\times\left\langle\pi^{-}\left|2 \pi^{0}\left(x_{1}\right) \frac{\sin 2 z\left(x_{1}\right)}{2 \sqrt{\pi^{2}\left(x_{1}\right)}} \sqrt{2}\left(\partial_{\mu} \pi^{0}\left(x_{2}\right) \pi^{+}\left(x_{2}\right)-\pi^{0}\left(x_{2}\right) \partial_{\mu} \pi^{+}\left(x_{2}\right)\right) \frac{\sin ^{2} y\left(x_{2}\right)}{y^{2}\left(x_{2}\right)}\right| 0\right\rangle\right\} .
\end{array}
$$

Here, as well as before, $\bar{U}_{p}, U_{n}$ stand for the proton and neutron wave amplitudes, and $M_{p}, M_{n}$ and $p_{p}, p_{n}$ are their masses and momenta. Let us recall the operator functions $\sin z / z, \cos z$, and so on , are treated as power series in square of the pion field operators $\varphi^{2}(x)=\pi^{2}(x)$, and the functions $y(x), z(x)$ have been introduced before in Sec. 2 , eqs. (21)-(23). As the expression (104) is a transition amplitude from pion vacuum $|n, 0\rangle$ to a state $\left\langle p, \pi^{-}\right|$containing one negative pion, $\pi^{-}$meson, the integrand in eq. (107) incorporates the matrix element $\left\langle\pi^{-}|\ldots| 0\right\rangle$ of a product of odd number of the pion field operators $\pi^{a}(x)$ depending on coordinates $x_{1}$ and $x_{2}$ of the vertexes $L^{1 W}\left(x_{1}\right)$ and $L^{1 W}\left(x_{2}\right)$ in eq. (104). According to the Wick-theorem, this matrix element is a sum of products of the matrix element $\left\langle\pi^{-}\left|\pi^{+}(x)\right| 0\right\rangle=u_{\pi r} \exp \left[i x q_{\pi}\right]$ times the vacuum expectations of the type

$$
\left\langle 0\left|\left(\partial_{\mu} \boldsymbol{\pi}\left(x_{1}\right) \boldsymbol{\pi}\left(x_{1}\right)\right) \boldsymbol{\pi}^{2 k}\left(x_{1}\right) \boldsymbol{\pi}^{2 m}\left(x_{2}\right)\right| 0\right\rangle,\left\langle 0\left|\boldsymbol{\pi}^{2 n}\left(x_{1}\right)\left(\partial_{\mu} \boldsymbol{\pi}\left(x_{2}\right) \boldsymbol{\pi}\left(x_{2}\right)\right) \boldsymbol{\pi}^{2 k}\left(x_{2}\right)\right| 0\right\rangle,
$$

or the matrix element $\left\langle\pi^{-}\left|\partial_{\mu} \pi^{+}(x)\right| 0\right\rangle=u_{\pi r}\left(i q_{\mu}\right) \exp \left[i x q_{\pi}\right]$ times the vacuum expectations of the form

$$
\left\langle 0\left|\boldsymbol{\pi}^{2 n}\left(x_{1}\right) \boldsymbol{\pi}^{2 m}\left(x_{2}\right)\right| 0\right\rangle .
$$


Every one of these vacuum expectations is in turn presented as a product of two kinds of vacuum expectations: the first one - a vacuum expectation of a product of the operators $\boldsymbol{\pi}^{2}(x)$, associated with one and the same vertex $L^{1 W}(x)$ and therefore depending on one single coordinate $x,\left\langle 0\left|\boldsymbol{\pi}^{2 n}(x)\right| 0\right\rangle=F_{0}$ (the closed thin-line circles in fig. 2), and the second one - a certain vacuum expectation from those set in eqs. (109), (108), depending on the coordinates $x_{1}$ and $x_{2}$ relating to the vertexes $L^{1 W}\left(x_{1}\right)$ and $L^{1 W}\left(x_{2}\right)$. (These vacuum expectations are pictured in fig. 2 by the bows attached to zigzag.) It is to keep in mind that $\left\langle 0\left|\left(\partial_{\mu} \boldsymbol{\pi}(x) \boldsymbol{\pi}(x)\right) \boldsymbol{\pi}^{2 k}(x)\right| 0\right\rangle=0$. With using the equations (34)-(36), the vacuum expectations $\left\langle 0\left|\boldsymbol{\pi}^{2 n}(x)\right| 0\right\rangle$ are expressed directly in terms of $\Delta(37)$ and does not depend on coordinates $x$ at all. Therefore the quantity $F_{0}$ can be brought out from under integrating over $x_{1}, x_{2}$ in eq. (107). The vacuum expectation of a pion field operators product, depending on coordinates $x_{1}$ and $x_{2}$, is expressed in terms of the quantities

$$
\begin{array}{r}
\left\langle 0\left|\mathcal{T} \pi^{\alpha}\left(x_{1}\right) \pi^{\beta}\left(x_{2}\right)\right| 0\right\rangle=\delta_{\alpha \beta} \int \frac{\mathrm{d}^{4} k}{(2 \pi)^{4}} \frac{i \exp \left[-i k\left(x_{1}-x_{2}\right)\right]}{k^{2}-m^{2}+i \epsilon}, \\
\left\langle 0\left|\mathcal{T} \partial_{\mu} \pi^{\alpha}\left(x_{1}\right) \pi^{\beta}\left(x_{2}\right)\right| 0\right\rangle=\delta_{\alpha \beta} \int \frac{\mathrm{d}^{4} k}{(2 \pi)^{4}} \frac{k_{\mu}}{k^{2}-m^{2}+i \epsilon},
\end{array}
$$

i.e. through the pion propagators and their derivatives. So it shows up to be function of the difference $x_{1}-x_{2}$. Denoting such vacuum expectations by $F\left(x_{1}-x_{2}\right)$, the eq. (107) would generally be represented as a sum of expressions of the form

$$
\begin{array}{r}
F_{0} i Q^{2} \int \mathrm{d}^{4} x_{1} \int \mathrm{d}^{4} x_{2} \int \frac{\mathrm{d}^{4} P}{(2 \pi)^{4}} \frac{\exp \left[-i P\left(x_{1}-x_{2}\right)\right]}{P^{2}-M_{W}^{2}+i \epsilon} \times \\
\times F\left(x_{1}-x_{2}\right) \exp \left[i x_{1}\left(p_{p}-p_{n}\right)+i x_{2} q_{\pi}\right]= \\
=F_{0} i Q^{2} \int \frac{\mathrm{d}^{4} k \tilde{F}(k)}{\left(p_{p}-p_{n}-k\right)^{2}-M_{W}^{2}} \delta\left(q_{\pi}+p_{p}-p_{n}\right) .
\end{array}
$$

As $p_{n}-p_{p} \ll M_{W}$, this difference is to be neglected here. The Furier-transform $\tilde{F}(k)$ of the function $F\left(x_{1}-x_{2}\right)$, as constructed by the functions (110), has a noticeable value at $k \ll M_{W}$, as $m \ll M_{W}$. Therefore eq. (111) can be transformed to

$$
\frac{i F_{0} Q^{2}}{-M_{W}^{2}} \int \mathrm{d}^{4} k \tilde{F}(k) \delta\left(p_{p}+q_{\pi}-p_{n}\right)=\frac{i F_{0} Q^{2}}{-M_{W}^{2}} \delta\left(p_{p}+q_{\pi}-p_{n}\right)(2 \pi)^{4} F(0),
$$

with accuracy $\sim m / M_{W}$. We would apparently receive the same result just putting $x_{1}=x_{2}$ in argument of the function $F$ in the expression (111). So, we can from the very first presume $x_{1}=x_{2}$ in calculating the matrix elements $\left\langle\pi^{-}|\ldots| 0\right\rangle$ of the products of pion field operators in the expression (107). As understood, due to $M_{W} \gg m, p_{p}, p_{n}, p_{\pi}$, integrating over the coordinates $x_{1}, x_{2}$ of two vertexes in eqs. (104), (107) is constricted into integrating over single variable. (In fig.2, this would be reflected by conflating two points $x_{1}$ and $x_{2}$.) Then we eventually obtain from eq. (107)

$$
\begin{array}{r}
\left\langle\pi^{-}, p\left|S_{p n \pi}^{W}\right| 0, n\right\rangle=\frac{\left|V_{u d}\right|^{2} G}{2 f} u_{\pi r}(2 \pi)^{4} \delta\left(p_{p}-p_{n}+q_{\pi}\right) \times \\
\times\left\{\left[g^{\prime} \bar{U}_{p} \gamma_{\mu} \gamma^{5} q_{\pi}^{\mu} U_{n}+\mathrm{Z}_{e} \bar{U}_{p} \gamma_{\mu} q_{\pi}^{\mu} U_{n}\right] \frac{1}{9}\left\langle 0\left|(1+2 \cos y)\left(2+\frac{\sin 2 y}{2 y}\right)\right| 0\right\rangle-\right.
\end{array}
$$




$$
\left.-\left[\mathrm{Z}_{e} g^{\prime} \bar{U}_{p} \gamma_{\mu} \gamma^{5} q_{\pi}^{\mu} U_{n}+\bar{U}_{p} \gamma_{\mu} q_{\pi}^{\mu} U_{n}\right] \frac{1}{3}\left\langle 0\left|\frac{\sin ^{3} y}{y}\right| 0\right\rangle\right\}
$$

The vacuum expectations herein are evaluated just alike the analogous quantities were calculated in the previous sections using eqs. (34)-(37), which results in

$$
\begin{array}{r}
\left\langle\pi^{-}, p\left|S_{p n \pi}^{W}\right| 0, n\right\rangle=\frac{\left|V_{u d}\right|^{2} G}{2 f}(2 \pi)^{4} \delta\left(p_{p}-p_{n}+q_{\pi}\right) u_{\pi r} \bar{U}_{p} \gamma_{\mu} q_{\pi}^{\mu} \times \\
\times\left\{\left[g^{\prime} \gamma^{5}+\mathrm{Z}_{e}\right] \frac{1}{9}\left[2+\exp (-1 / 4 P)\left(\frac{9}{2}-\frac{2}{P}+\frac{3}{2} \exp (-2 / P)\right)+\exp (-1 / P)\right]-\right. \\
\left.-\left[1+g^{\prime} \mathrm{Z}_{e} \gamma^{5}\right] \frac{1}{4} \exp (-1 / 4 P)(1-\exp (-2 / P))\right\} U_{n},
\end{array}
$$

where $P$ is defined by eq. (41). In the physical limit, that is at $d \rightarrow 4, \Delta \rightarrow \infty$ in the definition (37) (see Appendix A), and $Z_{e}=3, g^{\prime}=3 g_{A}$ accordingly eqs. (60), (67), one obtains

$$
\begin{aligned}
\left\langle\pi^{-}, p\left|S_{p n \pi}^{W}\right|\right. & 0, n\rangle=\frac{\left|V_{u d}\right|^{2} G}{2 f}(2 \pi)^{4} \delta\left(p_{p}-p_{n}+q_{\pi}\right) \frac{2}{3} \times \\
& \times\left\{g_{A} u_{\pi r} \bar{U}_{p} \gamma_{\mu} \gamma^{5} q_{\pi}^{\mu} U_{n}+u_{\pi r} \bar{U}_{p} \gamma_{\mu} q_{\pi}^{\mu} U_{n}\right\} .
\end{aligned}
$$

In the lowest $f^{2} \pi^{2}$-order, when $P^{-1} \rightarrow 0$, and $g^{\prime}=g_{A}$ accordingly eq. (66), $\mathrm{Z}_{e}=1$ accordingly eq. (60), the amplitude (114) reduces plainly to the expression (115) yet without the multiplier $2 / 3$. This last outcome would apparently be obtained from the eq. (104) if one treated therein immediately the hadron currents (50), (52) in the lowest $\varphi^{2}$-order:

$$
\mathcal{J}_{\varphi 5 \mu} \approx \frac{1}{2 f} \partial_{\mu} \varphi, \mathcal{J}_{N 5 \mu} \approx g_{A} \bar{N} \gamma^{\mu} \gamma^{5} \frac{\boldsymbol{\tau}}{2} N
$$

In this case, the amplitude $\left\langle\pi^{-}, p\left|S_{p n \pi}^{W}\right| 0, n\right\rangle$ would be depicted by the diagram in fig. 2, yet now without the thin lines.

To judge how significant $P$-parity violation is in the $n \rightarrow p+\pi^{-}$process (the $N N \pi$-vertex), we are to correlate the amplitudes (114), (115) with the amplitudes (98), (99) that describe this transition without violating $P$-parity. The first term in the expression (115), involving operator $\gamma^{5}$, does not violate $P$-invariance, yet causes only a modification of the parity-conserving pion-nucleon interaction amplitude, which is rather of no value. The second term, not including $\gamma^{5}$ operator, is obviously $P$-non-invariant. The total transition amplitude (106), with allowance for parity violating, is then written as

$$
\left\langle p, \pi^{-}\left|S_{n p \pi}^{t o t}\right| n, 0\right\rangle=(2 \pi)^{4} \delta\left(p_{p}-p_{n}+q_{\pi}\right) u_{\pi r} \bar{U}_{p} \gamma_{\mu} q_{\pi}^{\mu}\left(\gamma^{5} \frac{g_{A} \sqrt{2}}{2 F_{\pi}}+\frac{2}{3}\left|V_{u d}\right|^{2} G F_{\pi}\right) U_{n} .
$$

So, as a matter of fact, we are to compare the quantity $\left(\sqrt{2} g_{A} / 2 F_{\pi}\right)\left(\bar{U}_{p} \gamma_{\mu} \gamma^{5} q_{\pi}^{\mu} U_{n}\right)$, coming from the amplitude (99), with one $\left(2\left|V_{u d}\right|^{2} G F_{\pi} / 3\right)\left(\bar{U}_{p} \gamma_{\mu} q_{\pi}^{\mu} U_{n}\right)$ from the amplitude (115). Upon utilizing the generally known magnitude of the quantities $g_{A}, F_{\pi}, G$, we infer that the $P$-parity violating part constitutes the portion

$$
h^{W} \approx 0.75 \cdot 10^{-7} \frac{\left(\bar{U}_{p} \gamma_{\mu} q_{\pi}^{\mu} U_{n}\right)}{\left(\bar{U}_{p} \gamma_{\mu} \gamma^{5} q_{\pi}^{\mu} U_{n}\right)}
$$


of the parity conserving part of the transition amplitude. The total transition amplitude (117) would directly be obtained if we described the considered pion emission by the lagrangian (21) with replacing therein $\gamma^{5} \Rightarrow \gamma^{5}+0.75 \cdot 10^{-7} \mathrm{I}$.

When we were dealing with on-mass-shell nucleons (yet still an off-mass-shall pion), the amplitude (117) would reduce to

$$
\begin{array}{r}
\left\langle p, \pi^{-}\left|S_{n p \pi}^{t o t}\right| n, 0\right\rangle=-(2 \pi)^{4} \delta\left(p_{p}-p_{n}+q_{\pi}\right) \times \\
\times u_{\pi r} \bar{U}_{p}\left(\gamma^{5} \sqrt{2} g_{N N \pi}+\frac{2}{3}\left|V_{u d}\right|^{2} G F_{\pi}\left(M_{n}-M_{p}\right)\right) U_{n} .
\end{array}
$$

Then the P-parity violating part would constitute the portion

$$
h_{(O M S)}^{W} \approx 0.75 \cdot 10^{-7} \frac{\left(M_{n}-M_{p}\right)}{\left(M_{n}+M_{p}\right)} \approx 0.5 \cdot 10^{-10}
$$

of the total transition amplitude. So, in this case, parity violating is realized to be rather not discernible. With allowance for eq. (103), one would come to this transition amplitude (the $N N \pi$-vertex) (118) starting with the pion-nucleon interaction $\mathcal{L}_{N N \pi}(97)$, replaced $\gamma^{5} \Rightarrow \gamma^{5}+\mathrm{I} h_{(O M S)}^{W}$ therein. It is to emphasize once again that eq. (117) reduces to eq. (118) (alike eq. (98) to eq. (100) does) for the case of on-mass-shell (free) nucleons, but never for the nucleons embedded into nuclear matter (in nuclei).

In framework of the approach developed in refs. [22-24], the $P$-odd amplitude of the considered transition $n \rightarrow p+\pi^{-}$is due to the phenomenological interaction

$$
\mathcal{L}_{N N \pi}^{D}=\frac{h_{\pi}^{1}}{\sqrt{2}} \bar{N}[\tau \times \varphi]_{3} N
$$

added to the interaction (97) (conserving $P$-parity) that defines the first term in the amplitude (118). There exists a number of attempts [22-24] to evaluate this coupling $h_{\pi}^{1}$ using the basic quark-models techniques, but they all have encountered significant ambiguities. Their results could be presented in terms of an allowable range for $h_{\pi}^{1}$, accompanied by a "best value" representing the best guess for it. The interaction (120) causes the transition amplitude

$$
\begin{array}{r}
\left\langle p, \pi^{-}\left|S_{n p \pi}^{D}\right| n, 0\right\rangle=\frac{i h_{\pi}^{1}}{\sqrt{2}}\left\langle p, \pi^{-}\left|\int \bar{N}[\boldsymbol{\tau} \times \boldsymbol{\varphi}]_{3} N \mathrm{~d}^{4} x\right| n, 0\right\rangle= \\
=-h_{\pi}^{1}\left\langle p, \pi^{-}\left|\int \bar{N}\left(\tau^{+} \pi^{+}\right) N \mathrm{~d}^{4} x\right| n, 0\right\rangle=-(2 \pi)^{4} \delta\left(p_{p}+q_{\pi}-p_{n}\right) u_{\pi r} \bar{U}_{p} U_{n} h_{\pi}^{1} .
\end{array}
$$

Correlating this quantity with the second term in the amplitude (118), we obtain

$$
h_{\pi}^{1}=\frac{2}{3}\left|V_{u d}\right|^{2} G F_{\pi}\left(M_{n}-M_{p}\right) \approx 10^{-9} \approx g_{\pi} 0.026, \quad\left(g_{\pi}=3.8 \cdot 10^{-8}\right)
$$

which is much smaller than the "best guess" value asserted in ref. [22], as well as the others discussed in refs. [22-24]. However it still falls within the "reasonable range" estimated in ref. [22]. As one infers, inquiring into the contemporary issues explicated in ref. [24], a reliable $h_{\pi}^{1}$ value is not be thought to be definitively obtained from experimental results by now, in spite of the grate deal of efforts. 
Besides the process just examined (the charge pion emission), we are to discuss the neutral pion (of-mass-shell) emission : $n[p] \rightarrow n[p]+\pi^{0}$. The corresponding transition amplitude $\left\langle n, \pi^{0}\left|S_{n n \pi}^{W}\right| n, 0\right\rangle$ is determined by the interaction of eq. (81) in much the same way as $\left\langle p, \pi^{-}\left|S_{p n \pi}^{W}\right| n, 0\right\rangle$ was by the one of eq. (79). It is straightforward to ascertain the parity violating part of the amplitude $\left\langle n, \pi^{0}\left|S_{n n \pi}^{W}\right| n, 0\right\rangle$ vanishes, just because the initial and final nucleons have got the equal masses. This is in accordance with the general theorem [25] that is valid in the $C P$-conserving limit which we employ. Apparently, the process involving $\pi^{0}$ is eliminated with the phenomenological interaction (120).

\section{Conclusions.}

As understood, we have successively, step by step come to findings, relying on the general symmetry principles.

Sufficient trueness of the findings is ensured as we pursue the consistent way set forth. We describe the low-energy electro-weak transitions in a pion-nucleon system by the developed effective lagrangian (75), essentially nonlinear in pion field. All the parameters emerging in its deriving have been in due course associated with the observable data.

As based on the adoption of eqs. (9), (10), (14), the low-energy effective lagrangian (19) (as well as the one (75) ensuing from that) incorporates the one- and two-derivative terms only. Each additional derivative $\partial_{\mu} U$ would enter into the effective pion-nucleon lagrangian with the coefficient $\sim 1 / F_{\pi}$ (see, for instance, [1]). In evaluating the matrix elements of pion and nucleon interactions, this derivative would become a factor of the momentum $q$ transferred in interactions. Since we consider the low-energy processes in which the relation $q / F_{\pi} \ll 1$ is suggested, the allowance for one- and two-derivative terms in the effective lagrangian is sufficient with acceptable accuracy.

By the same reason of treating the low-energy processes, the fields interactions $L^{e m}, L^{1 W}, \mathcal{L}_{\varphi \varphi}, \mathcal{L}_{1 N \varphi}^{i n t}, \ldots$ themselves are allowed for at the lowest order in the calculations explicated in Sec. IV, V.

Strong interactions are assured to be adequately allowed for in the approach presented, which is based on the effective lagrangian essentially nonlinear in pion field. In our treatment, we are dealing with the infinite series in pion field. It is of crucial value that we have never restricted ourselves by allowance for a finite number of terms in the power series in $\varphi^{2}$, yet we summarize all this infinite series, in treating the considered processes. Just allowance for all the infinite series in $\varphi^{2}$ provides, as we have seen, to avoid occurrence of divergence in the developed treatment. In fine, we dare to assert that the plausible accuracy of our evaluations (in particular that of the eq. (122) ) is about a few tens of per-cents, at least.

All the calculations that have heretofore been discussed are restricted by allowance for the electroweak interaction in the lowest order. Certainly, to analyze the observed data with high accuracy $(\sim 1 \%$ or better), the radiative corrections are to be taken into consideration in studying the electro-weak 
processes. The approach developed would be of use in a simultaneous treatment of strong and electroweak interactions in these processes.

\section{Appendix A.}

To deal with the quantities akin to $\Delta(35), \Delta_{\mu \nu}(31)$, an idea is to generalize the dimensionality of the space from four (4) to a number $d$ (generally speaking, complex) which is called the regularizing parameter [12]. The integrands in eqs. (31), (35) are functions of $p^{2}=\eta^{\mu \nu} p_{\mu} p_{\nu}$, with $\eta^{\mu \nu}$ being a diagonal form with $(\nu-1)$ values +1 and one -1 . Modifying properly the integration measure over momenta, we rewrite $\Delta$ in $d$-dimensions in the Euclidean matric

$$
\begin{array}{r}
\Delta(d)=\frac{i \mu^{\epsilon}}{(2 \pi)^{d}} \int \frac{\mathrm{d}^{d} p}{p^{2}+m^{2}}= \\
=\frac{\mu^{\epsilon}}{2^{d} \pi^{d / 2}} \frac{1}{\Gamma(d / 2)} \int_{0}^{\infty} \frac{\mathrm{d}\left(p^{2}\right)\left(p^{2}\right)^{d / 2-1}}{p^{2}+m^{2}}, \quad \epsilon=d-4,
\end{array}
$$

where, as usual, $\mu$ is an auxiliary quantity having dimension of mass, $\Gamma$ is the Euler Gamma-function, and physical limit corresponds to $d \rightarrow 4$. As seen, an implementation of the dimensional regularization necessitates treating an integral of the type (Euler integral)

$$
I(d)=\int_{0}^{\infty} \frac{x^{d / 2-1}}{x+1} \mathrm{~d} x
$$

multiplied by $\left(m^{2}\right)^{d / 2-1}$. Separating, in the usual fashion, integer part of $d / 2$,

$$
d / 2=\nu_{d}+n_{d}, \text { with } n_{d}=0, \pm 1, \pm 2, \ldots, \text { and }+0 \leq \operatorname{Re} \nu_{d} \leq 1-0,
$$

the integral (A.2) is generally expedient to be presented in the form

$$
I_{n_{d}}\left(\nu_{d}\right)=\int_{0}^{\infty} \frac{x^{\nu_{d}+n_{d}-1}}{x+1} \mathrm{~d} x \equiv I(d) .
$$

Of course, for a given $d$ value, the number $n_{d}$ is dictated by eq. (A.3).

The function $I(d)=I_{n_{d}}\left(\nu_{d}\right)$ (A.2), (A.4) is clearly only sensible for

$$
+0 \leq \operatorname{Re} d / 2 \leq 1-0 \text {, i.e. for } n_{d}=0 .
$$

To deal with the function $I(d)\left(\right.$ A.2), (A.4) at a certain $n_{d} \neq 0$ corresponding to a given $d$ value, in particular, in a vicinity of the physical value, $d=4$, we are to resort to the analytic extension (continuation) of the function $I_{n_{d}=0}\left(\nu_{d}\right)$ to values $n_{d} \neq 0$ (see, for instance, refs. [26-29]).

For $n_{d}=0$ the analytic function $I(d)=I_{n_{d}=0}\left(\nu_{d}\right)$ is calculated as follows. To start with, we write

$$
I_{n_{d}=0}\left(\nu_{d}\right)=\int_{0}^{1} \mathrm{~d} x+\int_{1}^{\infty} \mathrm{d} x=I^{(1)}+I^{(2)} .
$$


For $+0 \leq x \leq 1-0$, the series expansion is valid

$$
\frac{x^{\nu_{d}-1}}{1+x}=\sum_{k=0}^{\infty}(-1)^{k} x^{\nu_{d}+k-1} \text {. }
$$

As the integral of this sum converges uniformly, both at $x=0$ and at $x=1$, integrating therm-by-therm holds true, which results in

$$
I^{(1)}=\sum_{k=0}^{\infty} \frac{(-1)^{k}}{\nu_{d}+k} .
$$

Upon setting $x=1 / z$, the integral $I^{(2)}$ results, in the same way, as

$$
I^{(2)}=\sum_{k=1}^{\infty} \frac{(-1)^{k}}{\nu_{d}-k}
$$

Recalling the common expansion of the function $\pi \operatorname{cosec}(\pi \nu)$ into simple fractions, the expression (A.6) proves eventually to be presented in the form

$$
I_{n_{d}=0}\left(\nu_{d}\right)=\pi(-1)^{m} \operatorname{cosec}\left[\pi\left(\nu_{d}+m\right)\right], \quad m=0, \pm 1, \pm 2, \ldots,+0 \leq \operatorname{Re} \nu_{d} \leq 1-0,
$$

which itself is analytic everywhere, except for the values $\operatorname{Re}\left(\nu_{d}+m\right)=0, \pm 1, \pm 2, \ldots$ In writing eq. (A.10), the prime relation is used: $\operatorname{cosec} \phi=(-1)^{n} \operatorname{cosec}(\phi+n \pi)$. With plainly implying $m=n_{d}$ in (A.10), this function (A.10) itself is obviously understood as analytical continuation (extension) [26-29] of the analytic function $I_{n_{d}=0}\left(\nu_{d}\right)$ to any $n_{d} \neq 0$ values, except for the values $\operatorname{Re}\left(\nu_{d}+n_{d}\right)=0, \pm 1, \pm 2, \ldots$. Indeed, both functions $I(d)$ and $\pi(-1)^{n_{d}} \operatorname{cosec}\left[\pi\left(\nu_{d}+n_{d}\right)\right]$ are analytic on the strip where they agree, $i . e$. at $n_{d}=0,+0 \leq \operatorname{Re}(d / 2) \leq 1-0$, but the last one is analytic on the wider domain.

Thus, upon extending, the required function

$$
I(d)=I_{n_{d}}\left(\nu_{d}\right)=\pi(-1)^{n_{d}} \operatorname{cosec}\left[\pi\left(\nu_{d}+n_{d}\right)\right]
$$

proves to be defined on all the $d$-plane, except for the points $\operatorname{Re}(d / 2)=0, \pm 1, \pm 2, \ldots$, where the function cosec itself possesses singularities.

It is expedient to clear up a behavior of the function $I(d)=I_{n_{d}}\left(\nu_{d}\right)$ at $d \rightarrow 0, \pm 1, \pm 2, \ldots$, especially at $\operatorname{Re} d \rightarrow 4$, the dimensionality of the physical space. Let $\operatorname{Re}(d / 2) \rightarrow 2-0$, that is $d$ tends to the physical value 4 from below. This corresponds to $\operatorname{Re} \nu_{d} \rightarrow 1-0$ at $n_{d}=1$ in the analytic extension $I_{n_{d}}\left(\nu_{d}\right)(\mathrm{A} .11)$ of the function $I_{n_{d}=0}\left(\nu_{d}\right)$. Then we arrive at

$$
(-1)^{1} \pi \operatorname{cosec}\left[\pi\left(\nu_{d}+1\right)\right] \longrightarrow+\infty, \text { when } \operatorname{Re} \nu_{d} \rightarrow 1-0
$$

Yet, if $d$ tends to the physical value $d=4$ from above, $\operatorname{Re}(d / 2) \rightarrow 2+0$, then in the analytical extension $I_{n_{d}}\left(\nu_{d}\right)$ (A.11) of the function $I_{n_{d}=0}\left(\nu_{d}\right)$ we have got $\operatorname{Re} \nu_{d} \rightarrow+0$ at $n_{d}=2$. This case corresponds to

$$
(-1)^{2} \pi \operatorname{cosec}\left[\pi\left(\nu_{d}+2\right)\right] \longrightarrow+\infty, \text { when } \operatorname{Re} \nu_{d} \rightarrow+0
$$


Eventually, we infer the function $\Delta(d)(37)$, being analytic on the $d$-plane, except for the points $\operatorname{Re} d / 2=$ $0, \pm 1, \pm 2, \ldots$, tends to $+\infty$, when $d$ tends to its physical value $d=4$, both from above and from below.

Amenably to the ordinary symmetry consideration, the relation is valid

$$
\int \mathrm{d}^{d} p \frac{p_{\alpha} p_{\beta}}{p^{2}+m^{2}}=\eta_{\alpha \beta} \frac{1}{d} \int \mathrm{d}^{d} p \frac{p^{2}}{p^{2}+m^{2}}
$$

which immediately yields eq. (38).

\section{References}

\section{References}

[1] J.F. Donoghue, E. Golowich and B.R. Holstein, Dynamics of the Standard Model. (Cambridge University Press, Cambridge, UK, 1994.)

[2] B. R. Holstain, Nucl. Phys. A 689, 135 (2001).

[3] V.Bernard and U-G. Meißner, Annual Rev. of Nucl. Part. Science, 57, 33 (2007); S.Scheres, In: “Advance in Nucl. Phys." 27, 201 (2003), ed. J.W. Negele and E. Vogt.

[4] C.N. Yang and R. Mills, Phys. Rev. 96, 191 (1954).

[5] P. Chang and F. Gürsey, Phys. Rev. 164, 1752 (1967);

F. Gürsey, Nuovo Cim. 16, 230 (1960);

F. Gürsey, Ann. Phys. (N.Y.) 12, 91 (1961).

[6] M.K. Volkov and V.N. Pervushin, Usp. Phys. Nauk. 120, 363 (1976) (in Russia). Soviet Physics Uspekhi, 19, iss. 11 (1977).

[7] M. K. Volkov and V. N Pervushin, Principle Nonlinear Quantum Theories, Dynamical Symmetries and Meson Physics. ("Atomizdat", Moscow, 1978.) (in Russia)

[8] D.V. Volkov, Part. and Nucl. 4, 3 (1973).

[9] S. Coleman, I. Wess and B. Summino, Phys. Rev. 177, 2239, 2247 (1969).

[10] M. Gell-Mann and Levy, Nuovo Cim. 16, 705 (1960);

S. Weinberg, Phys. Rev. Lett. 18, 188 (1967).

[11] G. Ecker and R.W. Honerkamp, Nucl. Phys. B 62, 509 (1973). 
[12] G. Leibbrant, Rev.Mod. Phys. 47, 849 (1975);

C. G. Bollini and J. J. Giambiagi, Nuovo Cim. B 12, 20 (1972);

C. G. Bollini and J. J. Giambiagi, Phys. Lett. 40B, 566 (1972);

J. F. Ashmore, Nuovo Cim. Lett., 4, 289 (1972);

G 't Hoft and W. Veltman, Nucl. Phys. B44, 189 (1972).

[13] G. H. Hardy, Divergent Series. (Oxford, UK, 1949.)

[14] S. Scherer and M. R. Schindler, Primer for Chiral Perturbation Theory, Lect. Notes Phys. 830. (Springer, Berlin, 2012)

[15] V.B. Beresteskii, E.M. Lifshits and L.P. Pitajevskii, Relativistic Quantum Theory, part I. (Pergamon, Oxford, 1971);

E.M. Lifshits and L.P. Pitajevskii, Relativistic Quantum Field Theory, part II. (Pergamon, Oxford, 1971.)

[16] S.L. Adler and R.F. Dashen, Current Algebras and Applications to Particle Physics. (Benjamin, Inc., New York-Amsterdam, 1968.)

[17] V. De Alfaro, S. Fubini, G. Furlan and G. Rosseti, Currents in Hadron Physiks. (American Elsevier Publishing Company, Inc., New York, 1973.)

[18] D.E. Groom et all., Rew. Part. Phys. (PDG), Eur. Phys. J. C 15, 1 (2000).

[19] E. D. Commins and P. H. Bucksbaum, Weak Interactions of Leptons and Quarks. (Cambridge University Press, 1983.)

[20] M. L. Goldberger and S. B. Treiman, Phys. Rev. 110, 1178 (1958).

[21] J.-W. Chen and X. Ji, Phys. Lett. B 501, 209. (2001);

E. Hernandes, J. Nieves and M. Valverde, Phys. Rev. D76, 033005 (2007);

P. F. Bedaque, M. J. Sawage, Phys. Rev. C62, 018501 (2000);

J.-W. Chen, T. D. Cohen, C. W. Kao, Phys. Rev. C64, 055206 (2001).

[22] B. Desplanques, J. E. Donoghue, and B. R. Holstein, Ann. Phys. (NY) 124, 449 (1980).

[23] V. M. Dubovik, S. V. Zenkin, Ann. Phys. (NY) 172, 100 (1986).

[24] G. B. Feldman, G. A. Crawford, J. Dubach, and B. R. Holstein, Phys. Rev. C43, 863 (1991);

N. Kaiser and Ulf-G. Meissner, Nucl. Phys. A510, 759 (1990);

B. R. Holstein, EPJ A 41, 279 (2009);

B. R. Holstein, Nucl. Phys. A844, 160 (2010);

W. C. Haxton and B. R. Holstein, Prog. Part. Nucl Phys. 71, 185 (2013). 
[25] G. Barton, Nuovo Cim. 19, 512 (1961).

[26] E. T. Whittaker and G. N. Watson, A Course of Modern Analysis, 4th ed. (Cambridge University Press, Cambridge, UK, 1990.)

[27] P. M. Morse and H. Feshbach, Methods of Theoretical Physics, Part 1. (New York: McGraw-Hill, USA, 1953.)

[28] K. Knopp, Theory of Functions, Part I. (New York: Dover, USA, 1996.)

[29] http://mathworld.wolfram.com/AnalyticContinuation.html; http://math.stackexchange.com/questions;

S.T.C. Siclos, http://damtp.cam.ac.uk/user/stcs/courses/fcm/sheets/sheet1.pdf 


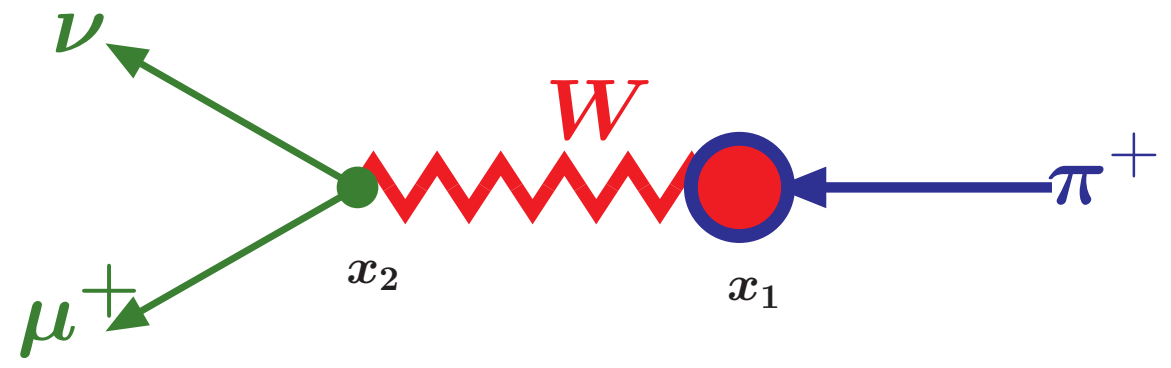

Fig. 1. Transition amplitude of the decay $\pi^{+} \rightarrow \mu^{+}+\nu$

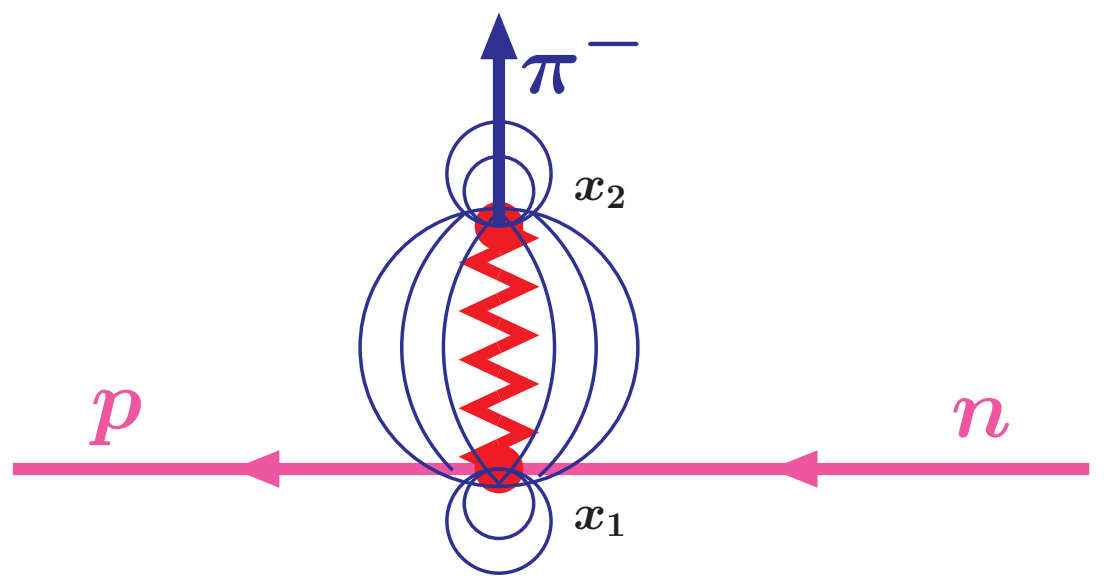

Fig. 2. The $n \rightarrow p+\pi^{-}$vertex with P-parity violation. 